K. TSUKADA

KODAI MATH. J.

19 (1996), 395-437

\title{
TOTALLY GEODESIC SUBMANIFOLDS OF RIEMANNIAN MANIFOLDS AND CURVATURE-INVARIANT SUBSPACES
}

\author{
KAZUMI TSUKADA
}

\section{Introduction}

An isometric immersion $\varphi: S \rightarrow M$ of a Riemannian manifold $S$ into another Riemannian manifold $M$ is called totally geodesic if the geodesics in $S$ are carried into geodesics in $M$. We call such a pair $(S, \varphi)$ a totally geodesic submanifold of $M$. Nevertheless, identifying $S$ and the image $\varphi(S)$, we often call the subset $\varphi(S)$ in $M$ a totally geodesic submanifold. Local problems are generally discussed in such a way. Among submanifolds of a Riemannian manifold, totally geodesic ones are fundamental.

Totally geodesic submanifolds of Riemannian symmetric spaces have been well investigated and it has been shown that they have beautiful and fruitful properties. In particular, due to the $\left(M_{+}, M_{-}\right)$-theory by B. Y. Chen and T. Nagano ([3]) this subject has made great progress. The author has a wish to understand well totally geodesic submanifolds of "general" Riemannian manifolds and obtained a few results in this paper.

We are concerned with three problems in this paper.

PROBLEM 1. For a given subspace $V$ in a tangent space $T_{p} M$, find good (or practical) criteria to conclude that there is a totally geodesic submanifold through $p$ whose tangent space at $p$ is $V$.

For this problem, we recall a theorem of E. Cartan is section 2 (Theorem 2.1 in this paper), which becomes a remarkable criterion if $M$ is a Riemannian symmetric space. That is, there is a totally geodesic submanifold whose tangent space is $V$ if and only if $V$ is a curvature-invariant subspace with respect to the Riemannian curvature tensor $R$, i.e.,

$$
R(x, y) z \in V \text { for any } x, y, z \in V .
$$

Is there such a criterion as above for a wider class of Riemannian manifolds? For example, for homogeneous Riemannian manifolds does there exist a finite number $d$ such that a condition similar to (1.1) for $R, \nabla R, \cdots, \nabla^{d} R$ implies the existence of totally geodesic submanifolds? In this paper we will show this for

Received April 1, 1996. 
naturally reductive homogeneous spaces (Theorem 2.3). And in section 7, with the same sense as the above question, we make "experiments" classifying totally geodesic surfaces in 3-dimensional Lie groups with left invariant Riemannian metrics (Theorem 7.2 and its observations).

In section 3 , to understand totally geodesic submanifolds in another point of view, we introduce a distribution $\mathfrak{D}$ on the Grassman bundle over a Riemannian manifold, which is an extension of the notion of geodesic spray on the unit sphere bundle. We show the relation between totally geodesic submanifolds and integral manifolds of $\mathfrak{D}$ (Theorem 3.1). Applying this relation, we consider the global existence problem of totally geodesic submanifolds (Theorem 3.5 and Theorem 3.6). These results are reformulations of a theorem of H. Reckziegel ([11]) and a theorem of R. Hermann ([7]).

It is a natural idea that "general" Riemannian manifolds will not have any $r$-dimensional totally geodesic submanifolds for $2 \leqq r \leqq n-1$. In fact, M. Spivak states in his book ([12], p. 39) as follows: "It seems rather clear that if one takes a Riemannian manifold $(N,\langle\rangle$,$) "at random", then it will not have any$ totally geodesic submanifolds of dimension $>1$. But I must admit that I don't know of any specific example of such a manifold". This yields our second problem.

Problem 2. Show that "general" Riemannian manifolds do not have any $r$-dimensional totally geodesic submanifolds for $2 \leqq r \leqq n-1$. Give specific examples of Riemannian manifolds which do not have any totally geodesic submanifolds.

Our results related to this problem are Theorem 5.4, examples after Proposition 6.6, and an observation of Theorem 7.2.

The following algebraic problem is motivated by Theorem 2.1 and it will play an important role in investigating totally geodesic submanifolds of Riemannian manifolds.

PROBLEM 3. Given a curvature tensor $R$ on $\boldsymbol{R}^{n}$, classify curvature-invariant subspaces with respect to $R$.

In sections 4 and 5, we study curvature-invariant subspaces. Let $G_{r}\left(\boldsymbol{R}^{n}\right)$ be the Grassmann manifold of $r$-dimensional subspaces in $\boldsymbol{R}^{n}$. How are $r$-dimensional curvature-invariant subspaces characterized in $G_{r}\left(\boldsymbol{R}^{n}\right)$ ? Some answers to this question are given as Theorem 4.1, Theorem 4.4, and Corollary 4.5. In the case of hyperplanes, such characterizations are stated as Proposition 4.6, Proposition 4.7, and Theorem 4.8. Theorem 4.8 is a variation of so-called axioms of planes. That is, the existence of many curvature-invariant subspaces implies that its curvature tensor is of constant sectional curvature. As an application of Theorem 4.8, a slight generation of Theorem 1 in B. Y. Chen ([2]) is obtained as Corollary 4.9. In section 5, we will classify curvature-invariant subspaces with respect to curvature tensors of special form (Propositions 5.1, 5.2 and 5.3). Applying these results, we construct examples of curvature tensors which don't 
have any $r$-dimensional curvature-invariant subspaces of $3 \leqq r \leqq n-1$ (Theorem 5.4). Comparing with Corollary 4.2, we see that it is different from 2-dimensional case

In sections 6 and 7 , we will classify totally geodesic submanifolds of specific and simple Riemannian manifolds. It also has the meaning of testing adequacy of our problems and our results. In section 6 , we classify totally geodesic submanifolds of hypersurfaces in $\boldsymbol{R}^{n+1}$ defined by the equations: $a_{1}\left(x^{1}\right)^{2}+a_{2}\left(x^{2}\right)^{2}$ $+\cdots+a_{n+1}\left(x^{n+1}\right)^{2}=1 \quad\left(a_{i} \neq 0 \quad i=1,2, \cdots, n+1\right) \quad$ or $\quad\left(x^{1}\right)^{3}+\left(x^{2}\right)^{3}+\cdots+\left(x^{n+1}\right)^{3}=1$ (Theorem 6.5 and Theorem 6.7). In section 7, Totally geodesic surfaces of 3-dimensional Lie groups with left invariant Riemannian metrics are classified (Theorem 7.2).

\section{Preliminaries-A theorem of E. Cartan on the existence of totally geodesic submanifolds of Riemannian manifolds}

In this section, we shall recall a theorem of E. Cartan on the existence of totally geodesic submanifolds of Riemannian manifolds and state related results.

Let $M$ be an $n(\geqq 3)$-dimensional Riemannian manifold. We introduce some notation. For a vector $u$ in the tangent space $T_{p} M$ at $p \in M$, we denote by $\gamma_{u}$ the geodesic through $p$ whose tangent vector at $p$ is $u$ and denote by $R_{u}(t)$ the $(1,3)$-tensor on $T_{p} M$ obtained by the parallel translation of the curvature tensor at $\gamma_{u}(t)$ along the geodesic $\gamma_{u}$. We define a $(1,2)$-tensor $r_{u}(t)$ on $T_{p} M$ by $r_{u}(t)(x, y)=R_{u}(t)(u, x) y, x, y \in T_{p} M$. Now we can give an answer to Problem 1 in section 1 . The following theorem is fundamental in our study, which is due to E. Cartan (cf. R. Hermann ([7])).

THEOREM 2.1. Let $V$ be a subspace of $T_{p} M$. Then the following three conditions are equivalent.

(1) There is a totally geodesic submanifold of $M$ through $p$ whose tangent space at $p$ is $V$.

(2) There is a positive number $\varepsilon$ such that for any unit vector $u \in V$ and any $t \in(-\varepsilon, \varepsilon)$, the following holds :

$$
R_{u}(t)(x, y) z \in V \quad \text { for any } x, y, z \in V .
$$

(3) There is a positive number $\varepsilon$ such that for any unit vector $u \in V$ and any $t \in(-\varepsilon, \varepsilon)$, the following holds:

$$
r_{u}(t)(x, y) \in V \text { for any } x, y \in V .
$$

Remark. The third condition in Theorem 2.1 is evidently weaker than the second one. The formulation of the third condition is due to K. Tojo ([13]).

The following is immediately seen. 
COROLLARY 2.2. Let $M$ be a real analytic Riemannian manifold and $V$ be a subspace of $T_{p} M$. Suppose that for $0 \leqq i<\infty$, the following holds:

$$
\left(\nabla^{i} R\right)(u, \cdots, u ; u, x, y) \in V \text { for } u, x, y \in V,
$$

where $\nabla^{i} R$ denotes the $i$-th covariant derivative of $R$. Then there is a totally geodesic submanifold of $M$ through $p$ whose tangent space at $p$ is $V$.

In view of Corollary 2.2, we have the following question: Does there exist a finite number $d$ such that the condition (2.3) for $0 \leqq i \leqq d$ implies the existence of a totally geodesic submanifold? We shall show that there exists such number for naturally reductive homogeneous spaces. Let $M=K / H$ be a naturally reductive homogeneous space with an $\operatorname{Ad}(H)$-invariant decomposition $\mathfrak{f}=\mathfrak{h}+\mathfrak{m}$ and with the $\operatorname{Ad}(H)$-invariant inner product $\langle$,$\rangle on \mathfrak{m}$ such that

$$
\left\langle[x, y]_{\mathfrak{m}}, z\right\rangle+\left\langle y,[x, z]_{\mathfrak{m}}\right\rangle=0 \text { for } x, y, z \in \mathfrak{m}
$$

(cf. S. Kobayashi and K. Nomizu [8] Chapter X). As usual, we identify $\mathfrak{m}$ with the tangent space $T_{o} M$ at the origin $o$ (=the coset $H$ ) of $M$. We denote by $\tau(h)$ the diffeomorphism of $M$ induced by $h \in K$. We put $\Lambda: \mathfrak{m} \times \mathfrak{m} \rightarrow \mathfrak{m}$ by $\Lambda(x) y=1 / 2[x, y]_{\mathfrak{m}}$ for $x, y \in \mathfrak{m}$. Then $(2.4)$ means that $\Lambda(x)$ is a skew-symmetric linear endomorphism of $(\mathfrak{m},\langle\rangle$,$) . Therefore e^{\Lambda(x)}$ is a linear isometry of $(\mathfrak{m},\langle\rangle$,$) . Since the Riemannian connection is a natural torsion-free connection$ on $K / H$, the following properties hold $([8]$, Ch. X):

(i) For each $x \in \mathfrak{m}$, the curve $\gamma_{x}(t)=\tau(\exp t x)(0)$ is a geodesic with $\gamma_{x}(0)=0$, $\gamma_{x}^{\prime}(0)=x$.

(ii) The parallel translation along $\gamma_{x}$ is given as follows;

$$
\tau(\exp t x)_{*} e^{-t \Lambda(x)}: T_{o} M(=\mathfrak{m}) \rightarrow T_{\gamma_{x}(t)} M .
$$

(iii) The $(1,3)$-tensor $R_{x}(t)$ on $\mathfrak{m}$ obtained by the parallel translation of the curvature tensor along $\gamma_{x}$ is given as follows:

$$
R_{x}(t)=e^{t \Lambda(x)} \cdot R_{o} .
$$

In the above, $R_{o}$ denotes the curvature tensor at the origin $o$ and $e^{t \Lambda(x)} \cdot$ denotes the action of $e^{t \Lambda(x)}$ on the space $\Re(\mathfrak{m})$ of curvature tensors on $\mathfrak{m}$. The formulas (ii) and (iii) are explicitly proved in K. Tojo ([13]). For each $u \in \mathfrak{m}$, we denote by $\Re_{u}$ the smallest subspace of $\Re(\mathfrak{m})$ which satisfies $R_{o} \in \Re_{u}$ and $\Lambda(u) \cdot \Re_{u} \subset \Re_{u}$. We define $d(u)$ by $d(u)=\operatorname{dim} \Re_{u}$. Trivially we have $d(u) \leqq \operatorname{dim} \mathfrak{R}(\mathfrak{m})$.

THEOREM 2.3. Let $M=K / H$ be a naturally reductive homogeneous space and $V$ be a subspace of $\mathfrak{m}\left(=T_{o} M\right)$. Suppose that for each unit vector $u \in V$, the following holds:

$$
\left(\nabla^{i} R\right)(u, \cdots, u ; u, x, y) \in V \text { for } 0 \leqq i \leqq d(u) \text {, and } x, y \in V .
$$


Then there is a totally geodesic submanifold of $M$ through o whose tangent space at $o$ is $V$.

Proof. At first, we recall the fundamental fact on the curve in the $m$-dimensional Euclidean space $\boldsymbol{R}^{m}$. Let $c: I \rightarrow \boldsymbol{R}^{m}$ be a curve defined on an open interval $I$ of $\boldsymbol{R}$ into $\boldsymbol{R}^{m}$. We say that $c$ has constant osculating rank $r$ if for all $t \in I$, its higher order derivatives $c^{\prime}(t), \cdots, c^{(r)}(t)$ are linearly independent and $c^{\prime}(t), \cdots, c^{(r+1)}(t)$ are linearly dependent in $\boldsymbol{R}^{m}$. It is a fundamental fact that if $c$ has constant osculating rank $r$, there exist smooth functions $a_{1}, \cdots, a_{r}$ : $I \rightarrow \boldsymbol{R}$ such that

$$
c(t)=c(0)+a_{1}(t) c^{\prime}(0)+\cdots+a_{r}(t) c^{(r)}(0) \text { for all } t \in I .
$$

We return to a naturally reductive homogeneous space $M$. For a unit vector $u \in V, R_{u}(t)=e^{t \Lambda(u)} \cdot R_{o}$ is a curve in $\Re(\mathfrak{m})$. Since $e^{t \Lambda(u)}$ is a 1-parameter subgroup of the group of linear isometries of $\Re(\mathfrak{m})$, the curve $R_{u}(t)$ has constant osculating rank $r$. Therefore we have

$$
R_{u}(t)=R_{u}(0)+a_{1}(t) R_{u}^{\prime}(0)+\cdots+a_{r}(t) R_{u}^{(r)}(0) .
$$

We note that $R_{u}^{(i)}(0)(x, y) z=\left(\nabla^{i} R\right)(u, \cdots, u ; x, y, z)$. Hence from the condition $\left(\nabla^{i} R\right)(u, \cdots, u ; u, x, y) \in V$ for $0 \leqq i \leqq r$ and $x, y \in V$, it follows that $r_{u}(t)(x, y) \in V$ for $x, y \in V$ and all $t$. On the other hand, since $R_{u}^{(i)}(0)=\Lambda(u)^{2} \cdot R_{o}, \Re_{u}$ coincides with the subspace of $\Re(\mathfrak{m})$ spanned by $R_{o}, \Lambda(u) \cdot R_{o}, \cdots, \Lambda^{(r)}(u) \cdot R_{0}$. In particular, we have $r=d(u)-1$ or $d(u)$.

\section{An extension of geodesic spray and the existence of totally geodesic submanifolds}

Let $M$ be an $n$-dimensional Riemannian manifold and $P$ be the bundle of orthonormal frames over $M$ with structure group $O(n)$. We fix an integer $r$, $1 \leqq r \leqq n-1$ and denote by $G_{r}\left(\boldsymbol{R}^{n}\right)=O(n) / O(r) \times O(n-r)$ the Grassmann manifold of $r$-dimensional subspaces of $\boldsymbol{R}^{n}$. Let $G_{r}(M)$ be the set of all $r$-dimensional subspaces of the tangent spaces at all points of $M$, i.e.,

$$
G_{r}(M)=\left\{(p ; V) \mid p \in M, V \text { is an } r \text {-dimensional subspace of } T_{p} M\right\} .
$$

Then it is the associated fibre bundle with $P$ with standard fibre $G_{r}\left(\boldsymbol{R}^{n}\right)$ which is expressed by $P \times_{O(n)} G_{r}\left(\boldsymbol{R}^{n}\right)$ or by $P / O(r) \times O(n-r)$. The bundle $G_{r}(M)$ is called the Grassmann bundle over $M$ and sometimes simply denote by $E$ in this section. We use the following symbols for associated projections:

$$
\begin{aligned}
& \pi: P \longrightarrow M, \\
& \pi_{E}: E \longrightarrow M, \\
& \mu: P \longrightarrow E .
\end{aligned}
$$


Here the projection $\mu$ is explicitly described as $\mu(u)=\left(\pi(u) ;\left\{u\left(e_{1}\right), \cdots, u\left(e_{r}\right)\right\}_{R}\right)$ for $u \in P$, where $\left\{e_{1}, \cdots, e_{n}\right\}$ is the natural basis of $\boldsymbol{R}^{n}$ and $\left\{u\left(e_{1}\right), \cdots, u\left(e_{r}\right)\right\}_{\boldsymbol{R}}$ denotes the subspace of $T_{\pi(u)} M$ spanned by orthonormal vectors $u\left(e_{1}\right), \cdots, u\left(e_{r}\right)$.

Let $\omega$ and $\theta$ be the Riemannian connection form and the canonical form on $P$, respectively; $\omega$ is an $\mathfrak{D}(n)$-valued 1 -form and $\theta$ is an $\boldsymbol{R}^{n}$-valued 1 -form. With respect to the natural basis $e_{1}, \cdots, e_{n}$ of $\boldsymbol{R}^{n}$, we write $\theta=\sum_{\imath=1}^{n} \theta^{2} e_{\imath}$. We define a distribution $\widetilde{D}$ of dimension $r$ on $P$ as follows: For each $u \in P$, we set

$$
\widetilde{\mathfrak{D}}_{u}=\left\{X \in T_{u} P \mid \omega(X)=0, \theta^{r+1}(X)=\cdots=\theta^{n}(X)=0\right\} .
$$

$\widetilde{D}_{u}$ is characterized as the subspace of $T_{u} P$ which satisfies $\widetilde{D}_{u} \subset \mathcal{H}_{u}$ and $\pi_{*}\left(\widetilde{\mathfrak{D}}_{u}\right)$ $=\left\{u\left(e_{1}\right), \cdots, u\left(e_{r}\right)\right\}_{R}$, where $\mathscr{H}_{u}$ denotes the horizontal subspace of $T_{u} P$ with respect to the Riemannian connection $\omega$. We denote by $R_{a}$ the right action on

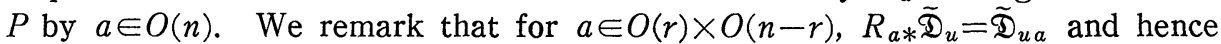
$\mu_{* u} \widetilde{\mathfrak{D}}_{u}=\mu_{* u a} \widetilde{\mathfrak{D}}_{u a}$. For each $\sigma=(p ; V) \in E=G_{r}(M)$, we choose $u \in P$ such that $\mu(u)=\sigma$ and set $\mathfrak{D}_{\sigma}=\mu_{* u} \widetilde{\mathfrak{D}}_{u}$. By the preceding remark it follows that $\mathfrak{D}_{\sigma}$ is independent of the choice of $u \in P$ such that $\mu(u)=\sigma$ and hence it is well-defined. Moreover we have $\pi_{E_{*}} \mathfrak{D}_{\sigma}=\pi_{*} \tilde{\mathfrak{D}}_{u}=\left\{u\left(e_{1}\right), \cdots, u\left(e_{r}\right)\right\}_{R}=V$. Thus a distribution $\mathfrak{D}$ of dimension $r$ on the Grassmann bundle $G_{r}(M)$ has been defined. We consider the case of $r=1$. Let $U M$ be the unit tangent bundle over $M$ and $G$ be the geodesic spray defined on $U M$. We denote by $\rho$ the bundle homomorphism of $U M$ onto $G_{1}(M)$ which is naturally defined. Then we have $\mathscr{D}_{\rho(x)}=\left\{\rho_{*} G_{x}\right\}_{\boldsymbol{R}}$ for all $x \in U M$. So we can view the distribution $\mathfrak{D}$ on $G_{r}(M)$ as an extended notion of geodesic spray.

We shall show that integral manifolds of the distribution $\mathscr{D}$ on $G_{r}(M)$ are in one to one correspondence with $r$-dimensional totally geodesic submanifolds of $M$. It is an analogous property to that of geodesic spray.

THEOREM 3.1. Let $f: S \rightarrow M$ be a totally geodesic imbedding of an $r$-dimensional connected Riemannian manifold $S$ into $M$ and $g$ be the mapping of $S$ into the Grassmann bundle $G_{r}(M)$ defined by $g(p)=\left(f(p) ; f_{*} T_{p} S\right)$ at $p \in S$. Then the image $g(S)$ is an integral manifold of the distribution $\mathfrak{D}$.

Conversely let $S$ be an integral manifold of $\mathfrak{D}$ on $G_{r}(M)$. The restriction of the projection $\pi_{E}: G_{r}(M) \rightarrow M$ to $S$ is a totally geodesic immersion of $S$ into $M$.

Proof. We will review the theory of submanifolds for the proof of Theorem 3.1. For this we follow [8] Chapter VII and describe submanifolds using the notion of frame bundles.

Let $f: S \rightarrow M$ be an imbedding of an $r$-dimensional connected manifold $S$ into $M$. We identify $S$ with the image $f(S)$ by $f$ if there is no danger of confusion; for any point $p$ of $S$ we shall denote $f(p) \in M$ by the same letter $p$. Then the tangent space $T_{p} S$ is a subspace of $T_{p} M$. Let $P \mid S$ and $E \mid S$ be the principal fibre bundle and its associated fibre bundle over $S$ induced by $f$ from the orthonormal frame bundle over $P$ and the Grassmann bundle $E=G_{r}(M)$ over $M$, respectively. $P \mid S$ and $E \mid S$ are given by 


$$
\begin{aligned}
& P \mid S=\{u \in P \mid \pi(u) \in S\}, \\
& E \mid S=\left\{\sigma \in E \mid \pi_{E}(\sigma) \in S\right\} .
\end{aligned}
$$

We denote by the same letter $j$ the injective homomorphism of $P \mid S$ into $P$ and that of $E \mid S$ into $E$. Associated with the imbedding $f$, the mapping $g: S \rightarrow E$ $=G_{r}(M)$ is defined by $g(p)=\left(f(p) ; f_{*} T_{p} S\right)\left(=\left(p ; T_{p} S\right)\right.$ under the preceding identification). Clearly we have $\pi_{E} \circ g=f$ and we can view $g$ as a cross section of $S$ into $E \mid S$. A frame $u \in P \mid S$ at $p \in S$ is said to be adapted if $\left\{u\left(e_{1}\right), \cdots\right.$, $\left.u\left(e_{r}\right)\right\}$ is a basis of $T_{p} S$ and hence $\left\{u\left(e_{r+1}\right), \cdots, u\left(e_{n}\right)\right\}$ is a basis of the normal space $T \stackrel{\perp}{p} S$. The set $Q$ of adapted frames forms a principal fibre bundle over $S$ with structure group $O(r) \times O(n-r) . \quad Q$ is the subbundle of $P \mid S$. It corresponds to the cross section $g$ of $E \mid S$ ([8] Chapter I Proposition 5.6). That is, the following holds: $Q=\{u \in P|S| \mu(u)=g(\pi(u))\}$, where $\pi$ and $\mu$ denote the projections of $P \mid S \rightarrow S$ and that of $P|S \rightarrow E| S$, respectively. We denote by $i$ the injective homomorphism of $Q$ into $P \mid S$. Then we have the following diagrams :
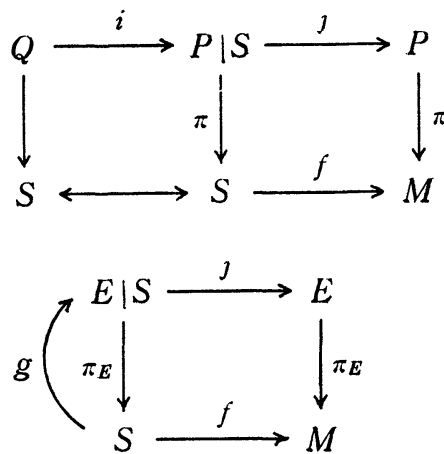

Let $\omega^{\prime}=j^{*} \omega$ be the connection on $P \mid S$ which is the restriction of the Riemannian connection $\omega$ on $P$. We note that the connection $\omega^{\prime}$ on $P \mid S$ is reducible to a connection on the subbundle $Q$ if and only if $S$ is a totally geodesic submanifold of $M$ (cf. [8] Chapter Vll Proposition 3.5 or Proposition 8.2). Let $\mathscr{H}^{\prime}$ be the horizontal distribution on $P \mid S$ defined by $\omega^{\prime}$. A distribution $D^{\prime}$ of dimension $r$ on $E \mid S$ is defined as follows. For each $\sigma \in E \mid S$ we choose $u \in P \mid S$ such that $\mu(u)=\sigma$ and set $\mathfrak{D}_{\sigma}^{\prime}=\mu_{*} \mathcal{H}_{u}^{\prime}$. Now we recall that $Q$ is the subbundle of $P \mid S$ corresponding to the cross section $g: S \rightarrow E \mid S$. It is known that the connection $\omega^{\prime}$ on $P \mid S$ is reducible to a connection on $Q$ if and only if at any point $p \in S, g_{*} T_{p} S=\mathfrak{D}_{g(p)}^{\prime}$ holds ([8] Chapter II Proposition 7.4). We see that $j_{*} \mathcal{H}_{u}^{\prime}=\widetilde{\mathfrak{D}}_{u}$ for each $u \in Q$. In fact, we have $\omega\left(j_{*} \mathcal{H}_{u}^{\prime}\right)=\omega^{\prime}\left(\mathcal{H}_{u}^{\prime}\right)=0$ and $\pi_{*} j_{*} \mathcal{H}_{u}^{\prime}=f_{*} \pi_{*} \mathcal{H}_{u}^{\prime}=f_{*} T_{\pi(u)} S=\left\{u\left(e_{1}\right), \cdots, u\left(e_{r}\right)\right\}_{R}$. Also we see that $j_{*} \mathfrak{D}_{g(p)}^{\prime}=$ $\mathfrak{D}_{g(p)}$ for each $p \in S$. In fact, choosing $u \in Q \cap \pi^{-1}(p)$, we have $\mu(u)=g(p)$ and $j_{*} \mathfrak{D}_{g(p)}^{\prime}=j_{*} \mu_{*} \mathcal{H}_{u}^{\prime}=\mu_{*} j_{*} \mathscr{H}_{u}^{\prime}=\mu_{*} \widetilde{\mathfrak{D}}_{u}=\mathfrak{D}_{g(p)}$. 
Now we shall prove Theorem 3.1. Let $f: S \rightarrow M$ be a totally geodesic imbedding. Then the connection $\omega^{\prime}$ on $P \mid S$ is reducible to a connection on the subbundle $Q$. From this it follows that for each point $p \in S, g_{*} T_{p} S=\mathfrak{D}_{\boldsymbol{g}(p)}^{\prime}$ holds. Since $j_{*} \mathfrak{D}_{g(p)}^{\prime}=\mathfrak{D}_{g(p)}$, the image $g(S)$ is an integral manifold of the distribution $\mathfrak{D}$.

Conversely let $S$ be an integral manifold of the distribution $\mathscr{D}$. We denote by $f$ the restriction of $\pi_{E}$ to $S$. Since for each $\sigma \in G_{r}(M), \pi_{E_{*}}$ restricted to the subspace $\mathfrak{D}_{\sigma}$ is injective, $f$ is an immersion. For each $\sigma=(p ; V) \in S \subset G_{r}(M)$, we have $f(\sigma)=\pi_{E}(\sigma)=p$ and $f_{*}\left(T_{p} S\right)=\pi_{E_{*}}\left(\mathfrak{D}_{\sigma}\right)=V$. Therefore the mapping $g: S \rightarrow G_{r}(M)$ associated with $f$ is exactly an inclusion mapping. We proceed backward and see that $f$ is totally geodesic.

Now we shall study the global existence problem of totally geodesic submanifolds. Our results stated here have been already obtained by H. Reckziegel ([11]) and R. Hermann ([7]). We will state them by another way, applying the preceding theorem.

We need to consider integral manifolds of a distribution which is not necessarily involutive. Even in this case, we can show the existence of maximal integral manifolds, exactly following the proof in the case of an involutive distribution (performed in C. Chevalley [4] Chapter III $\S$ VIII). Let $N$ be a manifold and $\mathscr{D}$ be a distribution of dimension $r$ on $N$. We don't assume that $\mathscr{D}$ is involutive. We state the following preparatory lemma which is essentially a consequence of 2.1 Affinity lemma in P. Dombrowski ([5]).

LEMMA 3.2. Let $S$ be an integral manifold of $\mathfrak{D}$. For each point $p \in S$, there exists a neighborhood $U$ of $p$ in $N$ which satisfies the following property. If $I$ is an open interval of $\boldsymbol{R}$ containing 0 and $\gamma: I \rightarrow N$ is a $C^{\infty}$-curve in $N$ such that $\gamma(0)=p, \gamma(I) \subset U$, and $\gamma^{\prime}(t) \in \mathscr{D}_{\gamma(t)}$ for any $t \in I$, then $\gamma(I)$ is contained in $S$ and $\gamma$ is a $C^{\infty}$-mapping of $I$ into $S$.

By this lemma, we obtain the following proposition, which corresponds to Chapter III \& VII Proposition 1 in [4].

Proposition 3.3. Let $S$ and $S^{\prime}$ be two integral manifolds of $\mathfrak{D}$ containing $a$ point $p$ in common. Then there exists an integral manifold containing $p$ such that it is an open submanifold of both $S$ and $S^{\prime}$.

After preparing this proposition, we can proceed by the same way as the case of an involutive distribution. Let $g$ be the subset of $N$ defined by

$$
g=\{p \in N \mid \text { there exists an integral manifold of } \mathfrak{D} \text { containing } p\} .
$$

We shall define a topology on $\mathcal{g}$. Let $\mathcal{O}$ be the family of those subsets of $\mathscr{g}$ which may be represented as unions of collections of integral manifolds of $\mathfrak{D}$. $\mathcal{O}$ satisfies the axioms of the family of open sets and hence provides a topology 
on 9 . Since for an arbitrary open subset $U$ in $N$ we have $U \cap \mathscr{G} \in \mathcal{O}, g$ equipped with this topology is a Hausdorff space. Let $S$ be a connected component of g. Then $S$ can canonically be equipped with the structure of an $r$-dimensional manifold and further it is an integral manifold of $\mathfrak{D}$. Thus we have the following.

THEOREM 3.4. Let $\mathfrak{D}$ be a distribution of dimension $r$ on $N$ which is not necessarily involutive and $g$ be the set of points of $N$ which are contained in integral manifolds of $\mathfrak{D}$. Then for each $p \in \mathcal{G}$, there exists a maximal integral manifold containing $p$, i.e., an integral manifold which is not a subset of any larger integral manifold. Any integral manifold containing $p$ is an open submanifold of this maximal integral manifold.

Applying Theorem 3.4, we shall show the existence of maximal totally geodesic submanifolds. H. Reckziegel in [11] proved the existence of maximally expanded isometric immersions with parallel second fundamental form. Our assertion is evidently a consequence of his result. In this paper we construct maximal totally geodesic submanifolds by another way. Let $\mathscr{D}$ be the distribution of dimension $r$ on the Grassmann bundle $G_{r}(M)$ which is defined in Theorem 3.1. We denote by $g$ the set of points of $G_{r}(M)$ which are contained in integral manifolds of $\mathfrak{D}$. By Theorem $3.1, g$ coincides with the set of elements $\sigma=(p ; V)$ in $G_{r}(M)$ which satisfy one of the equivalent three conditions in Theorem 2.1. Then we have the following.

THEOREM 3.5. For each $\sigma=(p ; V) \in \mathcal{G}$, there exists a maximal totally geodesic immersion $\phi: S \rightarrow M$ of an $r$-dimensional connected Riemannian manifold $S$ into $M$ in the following sense: Let $f: N \rightarrow M$ be any totally geodesic immersion of an $r$-dimensional connected Riemannian manifold $N$ into $M$ through $p$ whose tangent space at $p$ is $V$. Then there exists an isometric immersion $g: N \rightarrow S$ such that $\phi \circ g=f$.

Proof. By Theorem 3.4, we take a maximal integral manifold $S$ of $\mathfrak{D}$ containing $\sigma$. Let $\phi$ be the restriction to $S$ of the projection $\pi_{E}: G_{r}(M) \rightarrow M$. Then by Theorem $3.1 \phi$ is a totally geodesic immersion of $S$ into $M$. Let $f: N \rightarrow M$ be a totally geodesic immersion which satisfies the assumption of our theorem. We set $g: N \rightarrow G_{r}(M)$ as $g(q)=\left(f(q) ; f_{*} T_{q} N\right)$ for $q \in N$. For each $q \in N$, we take a connected neighborhood $U$ of $q$ such that $\left.f\right|_{U}: U \rightarrow M$ is imbedding. Then by Theorem $3.1 g(U)$ is an integral manifold of $\mathscr{D}$. Hence by Theorem 3.4, $g(U)$ is an open submanifold of some maximal integral manifold. From the connectedness of $N$, it follows that $g(N)$ is contained in $S$ and that $g: N \rightarrow S$ is an immersion. Clearly we have $\phi \circ g=f$. This implies that $g$ is an isometric immersion of $N$ into $S$.

From now on we assume that $M$ is a complete Riemannian manifold. We shall consider a necessary and sufficient condition that given a point $p \in M$ and 
an $r$-dimensional subspace $V$ of $T_{p} M$, there exists a complete totally geodesic submanifold of $M$ through $p$ whose tangent space at $p$ is $V$. Our statement is an another formulation of R. Hermann's result in [7]. We will prepare the notation. Let $\sigma=(p ; V) \in G_{r}(M)$ be given. For $v \in V$, we denote by $\gamma_{v}: \boldsymbol{R} \rightarrow M$ the geodesic of $M$ such that $\gamma_{v}(0)=p$ and $\gamma_{v}^{\prime}(0)=v$ and denote by $V(t)$ the subspace of $T_{r_{v}(t)} M$ obtained by parallel translating $V$ along $\gamma_{v}$ to the point $\gamma_{v}(t)$. Evidently we have $\gamma_{v}^{\prime}(t) \in V(t)$. We put $\sigma_{v}(t)=\left(\gamma_{v}(t) ; V(t)\right)$. Then $\sigma_{v}$ : $\boldsymbol{R} \rightarrow G_{r}(M)$ is a horizontal curve of $G_{r}(M)$ with respect to the Riemannian connection with $\sigma_{v}(0)=\sigma$. Moreover since $\pi_{E_{*}} \sigma_{v}^{\prime}(t)=\gamma_{v}^{\prime}(t) \in V(t)$, we have $\sigma_{v}^{\prime}(t) \in$ $\mathfrak{D}_{\sigma_{v}(t)}$. Using the notation, we formulate the following.

THEOREM 3.6. Let $M$ be a complete Riemannian manifold. For $\sigma=(p ; V)$ $\in G_{r}(M)$, the following two conditions are equivalent:

(1) There is a complete totally geodesic submanifold of $M$ through $p$ whose tangent space at $p$ is $V$.

(2) For all $v \in V$ and $t \in \boldsymbol{R}, \sigma_{v}(t) \in \mathcal{G}$, where $\mathcal{g}$ denotes the subset of $G_{r}(M)$ defined in Theorem 3.5.

Proof. The implication $(1) \rightarrow(2)$. Let $S$ be a complete Riemannian manifold and $f: S \rightarrow M$ be a totally geodesic immersion with a point $o \in S$ which satisfies $f(o)=p$ and $f_{*} T_{o} S=V$. For $v \in V$, we take $u \in T_{o} S$ which satisfies $f_{*} u=v$. Let $\gamma_{u}: R \rightarrow S$ be the geodesic of $S$ through $o$ whose tangent vector at $o$ is $u$. Then we have $f\left(\gamma_{u}(t)\right)=\gamma_{v}(t)$ and $f_{*} T_{\gamma_{u}(t)} S=V(t)$. Here $V(t)$ denotes the subspace of $T_{\gamma_{v}(t)} M$ obtained by parallel translating $V$ along $\gamma_{v}$ to the point $\gamma_{v}(t)$. Hence we see that $g\left(\gamma_{u}(t)\right)=\left(f\left(\gamma_{u}(t)\right) ; f_{*} T_{\gamma_{u}(t)} S\right)=\left(\gamma_{v}(t) ; V(t)\right)=\sigma_{v}(t)$. For each $t \in \boldsymbol{R}$, we take a connected neighborhood $U$ of $\gamma_{u}(t)$ in $S$ such that $\left.f\right|_{U}: U \rightarrow M$ is imbedding. Then by Theorem 3.1, $g(U)$ is an integral manifold of $D$ which contains $g\left(\gamma_{u}(t)\right)=\sigma_{v}(t)$. From this it follows that $\sigma_{v}(t) \in \mathcal{G}$.

The implication $(2) \rightarrow(1)$. By the assumption, we have $\sigma \in \mathcal{g}$. We take a maximal integral manifold $S$ of $\mathscr{D}$ containing $\sigma$. Then $\phi=\left.\pi_{E}\right|_{S}$ is a totally geodesic immersion of $S$ into $M$. We shall prove that $\sigma_{v}(t) \in S$ for any $v \in V$ and $t \in \boldsymbol{R}$. By Lemma 3.2 we see that for each $t_{0} \in \boldsymbol{R}$ there exists a positive number $\varepsilon$ such that $\sigma_{v}(t)$ for $\left|t-t_{0}\right|<\varepsilon$ are contained in a maximal integral manifold containing $\sigma_{v}\left(t_{0}\right)$. From the connectedness of $\boldsymbol{R}$, it follows that $\sigma_{v}(t) \in S$ for all $t \in \boldsymbol{R}$. Since $\phi\left(\sigma_{v}(t)\right)=\gamma_{v}(t), \sigma_{v}$ is a geodesic of $S$. Noticing that $\phi_{*}\left(\sigma_{v}^{\prime}(0)\right)$ $=\gamma_{v}^{\prime}(0)=v$ and that $\phi_{*_{\sigma}}$ is a linear isomorphism of $T_{\sigma} S$ onto $V$, we see that the exponential mapping $\exp _{\sigma}$ at $\sigma \in S$ is defined on all of $T_{\sigma} S$. Hence $S$ is a complete Riemannian manifold.

We define subsets $g^{(k)}$ and $\mathcal{G}_{k}$ of $G_{r}(M)$ for $k=0,1, \cdots$ as follows:

$$
\begin{aligned}
g^{(k)}=\left\{\sigma=(p ; V) \in G_{r}(M) \mid\right. & \left(\nabla^{k} R\right)\left(u_{1}, \cdots u_{k} ; x, y, z\right) \in V \\
& \text { for any } \left.u_{1}, \cdots, u_{k}, x, y, z \in V\right\}
\end{aligned}
$$




$$
\mathfrak{I}_{k}=\mathcal{g}^{(0)} \cap \mathcal{G}^{(1)} \cap \cdots \cap \mathcal{G}^{(k)} .
$$

Then $g^{(k)}$ and $g_{k}$ are closed subsets of $G_{r}(M)$. Evidently we see that

$$
\mathscr{g}_{0} \supset \mathcal{I}_{1} \supset \cdots \supset \mathcal{I}_{k} \supset \mathcal{G}_{k+1} \supset \cdots
$$

and that $\cap_{k=0}^{\infty} \mathcal{I}_{k} \supset \mathcal{G}$. Corollary 2.2 implies that $\cap_{k=0}^{\infty} \mathcal{I}_{k}=\mathscr{g}$ for a real analytic Riemannian manifold $M$. In particular $g$ is a closed subset of $G_{r}(M)$. By Theorem 2.3 we see that for a naturally reductive homogeneous space, there exists a finite integer $d$ such that $\mathcal{I}_{d}=\mathcal{g}$.

Applying Theorem 3.6, we have the following.

COROLlARY 3.7. Let $M$ be a real analytic complete Riemannian manifold. For $\sigma=(p ; V) \in \mathcal{g}$, there is a complete totally geodesic submanifold of $M$ through $p$ whose tangent space at $p$ is $V$.

Proof. We follow the notation of Theorem 3.6. For $v \in V$ let $\sigma_{v}: \boldsymbol{R} \rightarrow$ $G_{r}(M)$ be a curve defined in Theorem 3.6. We put $I=\left\{t \in \boldsymbol{R} \mid \sigma_{v}(t) \in g\right\}$. Since $g$ is a closed subset of $G_{r}(M)$ for a real analytic Riemannian manifold, $I$ is closed in $\boldsymbol{R}$. On the other hand, as it has been seen in the proof of Theorem 3.6, $I$ is open in $\boldsymbol{R}$. Since $\sigma_{v}(0)=\sigma \in \mathcal{G}, I$ contains 0 . Consequently $I$ coincides with $\boldsymbol{R}$. From Theorem 3.6, Corollary 3.7 is thereby proved.

\section{Characterizations of curvature-invariant subspaces}

Let $\boldsymbol{R}^{n}$ be an $n$-dimensional real vector space equipped with a standard inner product $\langle$,$\rangle . We denote by \Re(n)$ the space of curvature tensors over $\boldsymbol{R}^{n}$. Given a curvature tensor $R \in \Re(n)$, we call an $r$-dimensional subspace $V$ of $\boldsymbol{R}^{n}$ curvature-invariant with respect to $R$ if it holds that

$$
R(x, y) z \in V \text { for any } x, y, z \in V \text {. }
$$

Here we assume that $2 \leqq r \leqq n-1$ when we have no reference. Viewing Theorem 2.1, we recognize that it is an important problem for investigating totally geodesic submanifolds to classify curvature-invariant subspaces with respect to a given curvature tensor. In this section we shall study characterizations of curvature-invariant subspaces.

Let $G_{r}\left(\boldsymbol{R}^{n}\right)$ be the Grassmann manifold of $r$-dimensional subspaces of $\boldsymbol{R}^{n}$. We want criteria that $V \in G_{r}\left(\boldsymbol{R}^{n}\right)$ is curvature-invariant with respect to a given curvature tensor. In the case of $r=2$, we recall a result of R. S. Kulkarni. A curvature tensor $R$ defines the sectional curvature

$$
K(R): G_{2}\left(\boldsymbol{R}^{n}\right) \longrightarrow \boldsymbol{R}
$$

by $K(R)(V)=\left\langle R\left(e_{1}, e_{2}\right) e_{2}, e_{1}\right\rangle$, where $\left\{e_{1}, e_{2}\right\}$ is an orthonormal basis of $V$. The following has been shown by R.S. Kulkarni [9] as Corollary of Proposition 5.1. 
THEOREM 4.1. $V \in G_{2}\left(\boldsymbol{R}^{n}\right)$ is a curvature-invariant subspace with respect to a given curvature tensor $R$ if and only if $V$ is a critical point of $K(R)$.

Immediately the following two corollaries yield.

COROLLARY 4.2. For every curvature tensor $R$, there are always curvatureinvariant 2-dimensional subspaces with respect to $R$.

We note that in the higher dimensional case a different phenomenon from this occurs. See Theorem 5.4 in the next section. Cartan.

The following is well-known as the axiom of planes which is due to $E$.

COROLLARY 4.3. If every 2-dimensional subspace is curvature-invariant with respect to $R$, then the curvature tensor $R$ has constant sectional curvature.

We shall consider a generalization of Theorem 4.1 to the case of higher dimensional subspaces. First we represent the Grassmann manifold $G_{r}\left(\boldsymbol{R}^{n}\right)$ as Riemannian symmetric pair. The rotation group $S O(n)$ acts on $G_{r}\left(\boldsymbol{R}^{n}\right)$ transitively. Let $e_{1}, \cdots, e_{n}$ be a natural basis of $\boldsymbol{R}^{n}$ and $V_{o}=\left\{e_{1}, \cdots, e_{r}\right\}_{\boldsymbol{R}}$ be the $r$-dimensional subspace linearly spanned by $e_{1}, \cdots, e_{r}$. We define a mapping $\pi: S O(n) \rightarrow G_{r}\left(\boldsymbol{R}^{n}\right)$ by $\pi(a)=a \cdot V_{o}=\left\{a e_{1}, \cdots, a e_{r}\right\}_{\boldsymbol{R}}=\left\{a_{1}, \cdots, a_{r}\right\}_{\boldsymbol{R}}$, where $a=$ $\left(a_{1}, \cdots, a_{r}, a_{r+1}, \cdots, a_{n}\right) \in S O(n)$ is written as the row of column vectors $a_{1}, \cdots, a_{r}, a_{r+1}, \cdots, a_{n}$. The isotropy group at $V_{o} \in G_{r}\left(\boldsymbol{R}^{n}\right)$ consists of all matrices of the form

$$
\left(\begin{array}{ll}
\alpha & 0 \\
0 & \beta
\end{array}\right) \in S O(n) \text {, where } \alpha \in O(r) \text { and } \beta \in O(n-r) .
$$

Denoting this subgroup by $S(O(r) \times O(n-r))$, we have $G_{r}\left(\boldsymbol{R}^{n}\right)=S O(n) / S(O(r) \times$ $O(n-r))$. Hereafter denote simply by $G$ and $K, S O(n)$ and $S(O(r) \times O(n-r))$, respectively. We regard $G$ as the total space of the principal fibre bundle $G\left(G_{r}\left(\boldsymbol{R}^{n}\right), K\right)$ over $G_{r}\left(\boldsymbol{R}^{n}\right)$ with structure group $K$ and projection $\pi$. Let $g$ and $\mathfrak{f}$ be the Lie algebra and the Lie subalgebra of $g$ corresponding to $G$ and $K$, respectively. The complement $\mathfrak{p}$ of the canonical decomposition $\mathfrak{g}=\mathfrak{f}+\mathfrak{p}$ is given by

$$
\mathfrak{p}=\left\{\left(\begin{array}{cc}
0 & -{ }^{t} X \\
X & 0
\end{array}\right) \mid X \in M_{n-r, r}(\boldsymbol{R})\right\},
$$

where $M_{n-r, r}(\boldsymbol{R})$ denotes the space of all real matrices with $n-r$ rows and $r$ columns. The tangent space $T_{V_{o}} G_{r}\left(\boldsymbol{R}^{n}\right)$ is identified with $\mathfrak{p}$ by the differential $\pi_{*}$.

Let $F_{r}$ be the natural $r$-dimensional vector bundle over $G_{r}\left(\boldsymbol{R}^{n}\right)$. Namely $F_{r}$ is the vector subbundle of the trivial bundle $G_{r}\left(\boldsymbol{R}^{n}\right) \times \boldsymbol{R}^{n}$ defined as follows:

$$
F_{r}=\left\{(V ; v) \mid V \in G_{r}\left(\boldsymbol{R}^{n}\right), v \in V\right\} \subset G_{r}\left(\boldsymbol{R}^{n}\right) \times \boldsymbol{R}^{n} .
$$


$F_{r}$ can be viewed as a vector bundle with standard fibre $\boldsymbol{R}^{r}$ associated with the principal fibre bundle $G\left(G_{r}\left(\boldsymbol{R}^{n}\right), K\right)$ which corresponds to the Lie group homomorphism of $K$ onto $O(r)$ :

$$
\left(\begin{array}{ll}
\alpha & 0 \\
0 & \beta
\end{array}\right) \longmapsto \alpha
$$

In fact defining a mapping of $G \times \boldsymbol{R}^{r}$ onto $F_{r}$ by

$$
(a ; v) \in G \times \boldsymbol{R}^{r} \longrightarrow\left(a \cdot V_{o} ; \sum_{i=1}^{r} a_{i} v^{2}\right) \in F_{r} \subset G_{r}\left(\boldsymbol{R}^{n}\right) \times \boldsymbol{R}^{n},
$$

we have $G \times{ }_{K} \boldsymbol{R}^{r}=F_{r}$. In particular we can regard $a \in G$ as a linear isomorphism of $\boldsymbol{R}^{r}$ onto the fibre $\left(F_{r}\right)_{a \cdot V_{o}}$ at $a \cdot V_{o} \in G_{r}\left(\boldsymbol{R}^{n}\right)$. We introduce the invariant connection on the principal fibre bundle $G\left(G_{r}\left(\boldsymbol{R}^{n}\right), K\right)$ which corresponds to the canonical decomposition $g=\mathfrak{f}+\mathfrak{p}$. We denote by $D$ the covariant differentiation in $F_{r}$ defined by the connection. Let $\Gamma F_{r}$ be the space of sections of $F_{r}$ and $\exp t X$ be the 1-parameter subgroup of $G$ generated by $X \in \mathfrak{p}$. Then under the identification of $T_{V_{o}} G_{r}\left(\boldsymbol{R}^{n}\right)$ with $\mathfrak{p}$, we have the following formula:

$$
D_{X} \varphi=I_{n}\left(\left.\frac{d}{d t}\right|_{t=0}(\exp t X)^{-1} \varphi(\exp t X) \cdot v_{o}\right)
$$

for $\varphi \in \Gamma F_{r}$ and $X \in T_{V_{o}} G_{r}\left(\boldsymbol{R}^{n}\right) \cong \mathfrak{p}$. Here $I_{n}$ denotes the unit matrix. Moreover $\exp t X \in G$ is regarded as a linear isomorphism of $\boldsymbol{R}^{r}$ onto $\left(F_{r}\right)_{(\exp t X) \cdot v_{o}}$ and $(\exp t X)^{-1} \varphi(\exp t X) \cdot V_{o}$ is understood as a curve in $\boldsymbol{R}^{r}$.

Let $T_{3}^{1}\left(F_{r}\right)=F_{r}^{*} \otimes F_{r}^{*} \otimes F_{r}^{*} \otimes F_{r}$ be the vector bundle of type $(1,3)$ tensors of $F_{r}$. We shall define a mapping $\phi$ of $\Re(n)$ into the space $\Gamma T_{3}^{1}\left(F_{r}\right)$ of sections of $T_{3}^{1}\left(F_{r}\right)$. For $R \in \Re(n)$ and $V \in G_{r}\left(\boldsymbol{R}^{n}\right)$, we define a $(1,3)$-type tensor $\phi(R)_{V}$ on $\left(F_{r}\right)_{V} \cong V$ by restricting $R$ to $V$, i.e.,

$$
\phi(R)_{V}(x, y) z=\text { the } V \text {-component of } R(x, y) z \text {, for } x, y, z \in V \text {, }
$$

where we take the $V$-component with respect to the orthogonal decomposition $\boldsymbol{R}^{n}=V+V^{\perp}$. Evidently $\phi$ is a linear homomorphism of $\mathfrak{R}(n)$ into $\Gamma T_{3}^{1}\left(F_{r}\right)$. Moreover we see that $\phi$ is injective. In fact, if $\phi(R)_{V}=0$ for all $V \in G_{r}\left(\boldsymbol{R}^{n}\right)$, then $R$ has constant sectional curvature 0 and hence $R \equiv 0$ (Note that $2 \leqq r \leqq n-1$ ).

Now we show the following.

THEOREM 4.4. $V \in G_{r}\left(\boldsymbol{R}^{n}\right)$ is a curvature-invariant subspace with respect to a given curvature tensor $R$ if and only if $D \phi(R)=0$ at $V \in G_{r}\left(\boldsymbol{R}^{n}\right)$.

Proof. We can discuss the above assertion at $V_{o}=\left\{e_{1}, \cdots, e_{r}\right\}_{R}$ without loss of generality. Let $E_{\imath}^{j}$ be the $n \times n$ matrix such that the entry at the $i$-th column and the $j$-th row is 1 and other entries are all zero. We put $\mathcal{E}_{k}^{a}=E_{k}^{a}-E_{a}^{k}$ $(a=r+1, \cdots, n, k=1, \cdots, r)$. Then $\left\{\mathcal{E}_{k}^{a} ; a=r+1, \cdots, n, k=1, \cdots, r\right\}$ forms a basis of $\mathfrak{p}$. The 1-parameter subgroup $\exp t \mathcal{E}_{k}^{a}$ of $G$ generated by $\mathcal{E}_{k}^{a}$ is given as follows: Put 


$$
\exp t \mathcal{E}_{k}^{a}=\left(e_{1}(t), \cdots, e_{r}(t), e_{r+1}(t), \cdots, e_{n}(t)\right)
$$

as the row of column vectors $e_{1}(t), \cdots, e_{r}(t), e_{r+1}(t), \cdots, e_{n}(t)$. Then we have

$$
\begin{aligned}
& e_{i}(t)=e_{\imath} \text { for } i \neq k, a \\
& e_{k}(t)=\cos t e_{k}+\sin t e_{a} \\
& e_{a}(t)=-\sin t e_{k}+\cos t e_{a} .
\end{aligned}
$$

For $1 \leqq i_{1}, i_{2}, i_{3}, i_{4} \leqq r$, we see that

$$
\begin{aligned}
& \left\langle\left(\exp t \mathcal{E}_{k}^{a}\right)^{-1} \phi(R)_{\left(\exp t \mathcal{E}_{k}^{a}\right) \cdot v_{o}}\left(e_{i_{1}}, e_{i_{2}}\right) e_{\imath_{3}}, e_{i_{4}}\right\rangle \\
= & \left\langle R\left(e_{i_{1}}(t), e_{i_{2}}(t)\right) e_{i_{3}}(t), e_{i_{4}}(t)\right\rangle .
\end{aligned}
$$

The formula (4.2) implies that

$$
\begin{aligned}
& \left\langle\left(D_{\mathcal{E}_{k}^{a}} \phi(R)\right)\left(e_{i_{1}}, e_{i_{2}}\right) e_{i_{3}}, e_{i_{4}}\right\rangle \\
= & \left.\frac{d}{d t}\right|_{t=0}\left\langle R\left(e_{i_{1}}(t), e_{i_{2}}(t)\right) e_{i_{3}}(t), e_{i_{4}}(t)\right\rangle \\
= & \delta_{i_{1} k}\left\langle R\left(e_{a}, e_{i_{2}}\right) e_{i_{3}}, e_{i_{4}}\right\rangle+\delta_{i_{2} k}\left\langle R\left(e_{i_{1}}, e_{a}\right) e_{i_{3}}, e_{i_{4}}\right\rangle \\
& +\delta_{i_{3} k}\left\langle R\left(e_{i_{1}}, e_{i_{2}}\right) e_{a}, e_{i_{4}}\right\rangle+\delta_{i_{4} k}\left\langle R\left(e_{i_{1}}, e_{i_{2}}\right) e_{i_{3}}, e_{a}\right\rangle .
\end{aligned}
$$

Therefore if $V_{o}$ is a curvature-invariant subspace with respect to $R$, we have $D \phi(R)=0$ at $V_{o}$. Conversely suppose that $D \phi(R)=0$ at $V_{0}$. We devide it into the three cases according to the values of $r$.

Case $1, r \geqq 4$. For $1 \leqq i_{1}, i_{2}, i_{3} \leqq r$, we choose $k$ such that $1 \leqq k \leqq r$ and $k \neq$ $i_{1}, i_{2}, i_{3}$. For each $a(r+1 \leqq a \leqq n)$, we have

$$
0=\left\langle\left(D_{\varepsilon_{k}}^{a} \phi(R)\right)\left(e_{i_{1}}, e_{\imath_{2}}\right) e_{i_{3}}, e_{\imath_{k}}\right\rangle=\left\langle R\left(e_{i_{1}}, e_{\imath_{2}}\right) e_{i_{3}}, e_{a}\right\rangle .
$$

Therefore we see that $V_{o}$ is curvature-invariant with respect to $R$.

Case 2, $r=3$. For each $a(4 \leqq a \leqq n)$, we get

$$
\begin{aligned}
0 & =\left\langle\left(D_{\varepsilon_{1}^{a}}^{a} \phi(R)\right)\left(e_{1}, e_{2}\right) e_{3}, e_{1}\right\rangle \\
& =\left\langle R\left(e_{a}, e_{2}\right) e_{3}, e_{1}\right\rangle+\left\langle R\left(e_{1}, e_{2}\right) e_{3}, e_{a}\right\rangle \\
& =\left\langle R\left(e_{1}, e_{3}\right) e_{2}, e_{a}\right\rangle+\left\langle R\left(e_{1}, e_{2}\right), e_{3}, e_{a}\right\rangle
\end{aligned}
$$

and similarly $\left\langle R\left(e_{2}, e_{3}\right) e_{1}, e_{a}\right\rangle+\left\langle R\left(e_{2}, e_{1}\right) e_{3}, e_{a}\right\rangle=0$. Therefore we have

$$
\begin{aligned}
0 & =\left\langle R\left(e_{1}, e_{2}\right) e_{3}, e_{a}\right\rangle+\left\langle R\left(e_{2}, e_{3}\right) e_{1}, e_{a}\right\rangle+\left\langle R\left(e_{3}, e_{1}\right) e_{2}, e_{a}\right\rangle \\
& =\left\langle R\left(e_{1}, e_{2}\right) e_{3}, e_{a}\right\rangle-\left\langle R\left(e_{2}, e_{1}\right) e_{3}, e_{a}\right\rangle-\left\langle R\left(e_{2}, e_{1}\right) e_{3}, e_{a}\right\rangle \\
& =3\left\langle R\left(e_{1}, e_{2}\right) e_{3}, e_{a}\right\rangle .
\end{aligned}
$$

The others are same. Hence we see that $V_{o}$ is curvature-invariant with respect to $R$. 
Case $3, r=2$. For each $a(3 \leqq a \leqq n)$, we get

$$
\begin{aligned}
0 & =\left\langle\left(D_{\mathcal{E}_{1}^{a}} \phi(R)\right)\left(e_{1}, e_{2}\right) e_{2}, e_{1}\right\rangle \\
& =\left\langle R\left(e_{a}, e_{2}\right) e_{2}, e_{1}\right\rangle+\left\langle R\left(e_{1}, e_{2}\right) e_{2}, e_{a}\right\rangle \\
& =2\left\langle R\left(e_{1}, e_{2}\right) e_{2}, e_{a}\right\rangle .
\end{aligned}
$$

Therefore we see that $V_{o}$ is curvature-invariant with respect to $R$.

Given a curvature tensor $R \in \Re(n)$, we can define various functions on $G_{r}\left(\boldsymbol{R}^{n}\right)$. For example, we have the following:

$$
\begin{aligned}
& \tau(\phi(R))(V)=\sum_{\imath, j=1}^{r}\left\langle R\left(\tilde{e}_{\imath}, \tilde{e}_{j}\right) \tilde{e}_{j}, \tilde{e}_{\imath}\right\rangle \\
& \|\rho(\phi(R))\|^{2}(V)=\sum_{\imath, j, k, l=1}^{r}\left\langle R\left(\tilde{e}_{\imath}, \tilde{e}_{k}\right) \tilde{e}_{k}, \tilde{e}_{j}\right\rangle\left\langle R\left(\tilde{e}_{\imath}, \tilde{e}_{l}\right) \tilde{e}_{l}, \tilde{e}_{j}\right\rangle \\
& \|\phi(R)\|^{2}(V)=\sum_{\imath_{1}, 2_{2}, \imath_{3}, l_{4}=1}^{r}\left\langle R\left(\tilde{e}_{i_{1}}, \tilde{e}_{\imath_{2}}\right) \tilde{e}_{\imath_{3}}, \tilde{e}_{i_{4}}\right\rangle\left\langle R\left(\tilde{e}_{\imath_{1}}, \tilde{e}_{\imath_{2}}\right) \tilde{e}_{i_{3}}, \tilde{e}_{\imath_{4}}\right\rangle
\end{aligned}
$$

where $\left\{\tilde{e}_{1}, \cdots, \tilde{e}_{r}\right\}$ denotes an orthonormal basis of $V$.

Using Theorem 4.4, we can prove the following.

COROLlARY 4.5. If $V \in G_{r}\left(\boldsymbol{R}^{n}\right)$ is a curvature-invariant subspace with respect to $R$, then $V$ is a critical point of functions $\tau(\phi(R)),\|\rho(\phi(R))\|^{2},\|\phi(R)\|^{2}, \cdots$.

In the rest of this section, we shall discuss the case of $r=n-1$, i.e., hyperplanes. By simple computation, we obtain the following which will be used in section 7 .

Proposition 4.6. If $V \in G_{n-1}\left(\boldsymbol{R}^{n}\right)$ is a curvature-invariant hyperplane with respect to a curvature tensor $R$, then the orthogonal complement $V^{\perp}$ is an eigenspace of its Ricci curvature $\rho$. When $n=3$, the converse also is true.

Proof. We take an orthonormal basis $\left\{\tilde{e}_{1}, \cdots, \tilde{e}_{n}\right\}$ of $\boldsymbol{R}^{n}$ such that $V$ is linearly spanned by $\tilde{e}_{1}, \cdots, \tilde{e}_{n-1}$. Since

$$
\rho\left(\tilde{e}_{n}, \tilde{e}_{j}\right)=\sum_{\imath=1}^{n-1}\left\langle R\left(\tilde{e}_{j}, \tilde{e}_{\imath}\right) \tilde{e}_{\imath}, \tilde{e}_{n}\right\rangle \text { for } j=1, \cdots, n-1,
$$

the first part of the above proposition holds.

When $n=3$, let $\left\{\tilde{e}_{1}, \tilde{e}_{2}, \tilde{e}_{3}\right\}$ be an orthonormal basis of $\boldsymbol{R}^{3}$ such that $\tilde{e}_{3}$ is an eigenvector of the Ricci tensor $\rho$. Since

and

$$
0=\rho\left(\tilde{e}_{3}, \tilde{e}_{1}\right)=\left\langle R\left(\tilde{e}_{1}, \tilde{e}_{22}\right) \tilde{e}, \tilde{e}_{3}\right\rangle
$$

$$
0=\rho\left(\tilde{e}_{3}, \tilde{e}_{2}\right)=\left\langle R\left(\tilde{e}_{2}, \tilde{e}_{1}\right) \tilde{e}_{1}, \tilde{e}_{3}\right\rangle,
$$


$V=\left\{\tilde{e}_{1}, \tilde{e}_{2}\right\}_{R}$ is curvature-invariant with respect to $R$.

Let $\Lambda^{2} \boldsymbol{R}^{n}$ be the space of 2-vectors of $\boldsymbol{R}^{n}$. For a given curvature tensor $R$, we define a symmetric endomorphism $\hat{R}$ on $\Lambda^{2} \boldsymbol{R}^{n}$, which is called a curvature operator. Namely put

$$
\langle\hat{R}(x \wedge y), u \wedge v\rangle=\langle R(x, y) u, v\rangle \text { for } x, y, u, v \in \boldsymbol{R}^{n}
$$

(cf. J.P. Bourguignon and H. Karcher [1]). The existence of curvature-invariant hyperplanes has an effect on the spectral data of the curvature operator $\hat{R}$. Namely the following holds.

PROPOSITION 4.7. Let $V \in G_{n-1}\left(\boldsymbol{R}^{n}\right)$ be a curvature-invariant hyperplane with respect to a curvature tensor $R$ and $\xi$ be a unit vector of $\boldsymbol{R}^{n}$ which is orthogonal to $V$. Then there is an orthonormal basis $\left\{\tilde{e}_{1}, \cdots, \tilde{e}_{n-1}\right\}$ of $V$ such that $\xi \wedge \tilde{e}_{2}$ $(i=1, \cdots, n-1)$ are eigenvectors of the curvature operator $\hat{R}$. In particular the curvature operator $\hat{R}$ has at least $n-1$ decomposable eigenvectors which are linearly independent.

Proof. Let $V$ and $\xi$ be the ones which satisfy the condition in Proposition 4.7. For $R$ and $\xi$, we define a symmetric endomorphism $\widetilde{R}_{\xi}$ of $\boldsymbol{R}^{n}$ by $\left\langle\widetilde{R}_{\xi}(x), y\right\rangle$ $=\langle R(\xi, x) \xi, y\rangle$ for $x, y \in \boldsymbol{R}^{n}$. Evidently we have $\widetilde{R}_{\xi}(V) \subset V$. We take an orthonormal basis $\left\{\tilde{e}_{1}, \cdots, \tilde{e}_{n-1}\right\}$ in $V$ such that $\tilde{e}_{\imath}(i=1, \cdots, n-1)$ are eigenvectors of $\widetilde{R}_{\xi}$, i.e., $\widetilde{R}_{\xi}\left(\tilde{e}_{2}\right)=\lambda_{i} \tilde{e}_{\imath}$. Then we see that $\xi \wedge \tilde{e}_{\imath}(i=1, \cdots, n-1)$ are also eigenvectors of the curvature operator $\hat{R}$ with eigenvalues $\lambda_{2}$. In fact, we get

and

$$
\left\langle\hat{R}\left(\xi \wedge \tilde{e}_{\imath}\right), \xi \wedge \tilde{e}_{j}\right\rangle=\left\langle R\left(\xi, \tilde{e}_{\imath}\right) \xi, \tilde{e}_{j}\right\rangle=\left\langle\tilde{R}_{\xi}\left(\tilde{e}_{\imath}\right), \tilde{e}_{\jmath}\right\rangle=\lambda_{i} \delta_{i j}
$$

$$
\left\langle\hat{R}\left(\xi \wedge \tilde{e}_{\imath}\right), \tilde{e}_{\jmath} \wedge \tilde{e}_{k}\right\rangle=\left\langle R\left(\xi, \tilde{e}_{\imath}\right) \tilde{e}_{\jmath}, \tilde{e}_{k}\right\rangle=\left\langle R\left(\tilde{e}_{k}, \tilde{e}_{j}\right) \tilde{e}_{\imath}, \xi\right\rangle=0 \text {. }
$$

Applying Proposition 4.7, we obtain the following which is an analogous result to the axiom of planes.

THEOREM 4.8. Let $H$ be the connected Lie subgroup of $S O(n)$ which acts irreducibly on $\boldsymbol{R}^{n}$ and $R$ be an invariant curvature tensor by the action of $H$. If there is a curvature-invariant hyperplane $V \in G_{n-1}\left(\boldsymbol{R}^{n}\right)$ with respect to $R$, then $R$ has constant sectional curvature.

Proof. Let $g \mathfrak{g}(n)$ be the Lie algebra which corresponds to $S O(n)$ and $\mathfrak{h}$ be the Lie subalgebra of $\mathfrak{g} \mathfrak{p}(n)$ which corresponds to $H$. By the assumption, $\mathfrak{h}$ acts irreducibly on $\boldsymbol{R}^{n}$. Let $V \in G_{n-1}\left(\boldsymbol{R}^{n}\right)$ be a curvature invariant hyperplane with respect to $R$ and $\xi$ be a unit vector of $\boldsymbol{R}^{n}$ which is orthogonal to $V$. Let $V=V_{1}+\cdots+V_{s}$ be the orthogonal decomposition of $V$ into the eigenspaces of $\tilde{R}_{\xi}$ and $\lambda_{2}(i=1, \cdots, s)$ be the eigenvalues of $\widetilde{R}_{\xi}$ on the eigenspaces $V_{\imath}$. It is assumed that $\lambda_{1}, \cdots, \lambda_{s}$ are mutually distinct. From Proposition 4.7, it follows 
that $\hat{R}(\xi \wedge v)=\lambda_{i} \xi \wedge v$ for $v \in V_{\imath}$. Now we shall prepare the following lemma.

LEMMA. For each $X \in \mathfrak{h}$ and $v \in V_{\imath}$, the following formulas hold:

(1) $\hat{R}(\xi \wedge X v)=\lambda_{i} \xi \wedge X v$,

(2) $\hat{R}(X \xi \wedge v)=\lambda_{2} X \xi \wedge v$.

Proof of Lemma. Let $\exp t X$ be the 1-parameter subgroup of $H$ generated by $X \in \mathfrak{h}$. Since the curvature tensor $R$ is invariant by the action of $H$, we have $\hat{R} \circ \exp t X=\exp t X \circ \hat{R}$ on $\Lambda^{2} \boldsymbol{R}^{n}$. Hence

$$
\hat{R}(\exp t X(\xi \wedge v))=\exp t X(\hat{R}(\xi \wedge v))=\lambda_{2} \exp t X(\xi \wedge v) .
$$

Differentiating this equation at $t=0$, we have

$$
\hat{R}(X \xi \wedge v+\xi \wedge X v)=\lambda_{i}(X \xi \wedge v+\xi \wedge X v) .
$$

We note that $\hat{R}\left(\Lambda^{2} V\right) \subset \Lambda^{2} V$ and $\hat{R}(\xi \wedge V) \subset \xi \wedge V$, since $V$ is curvature-invariant with respect to $R$. Since $X \xi \in V$, we obtain $\hat{R}(\xi \wedge X v)=\lambda_{i} \xi \wedge X v$ and $\hat{R}(X \xi \wedge v)$ $=\lambda_{i} X \xi \wedge v$. Thus Lemma has been proved.

By Lemma (1), we see that for each eigenspace $V_{\imath}$ and any $X \in \mathfrak{h}, X\left(V_{\imath}\right) \subset$ $V_{i}+\boldsymbol{R} \cdot \xi$. If $X \xi=0$ for any $X \in \mathfrak{h}$, it contradicts to the irreducibility of $\mathfrak{h}$. Therefore there exists some $X_{o} \in \mathfrak{h}$ such that $X_{o} \xi \neq 0$. Accordingly, for some $i \in\{1, \cdots, s\}$ and $v_{o} \in V_{\imath}$ we have $\left\langle X_{o} \xi, v_{0}\right\rangle \neq 0$. For this $i$, we shall show that $X \xi \in V_{\imath}$ for any $X \in \mathfrak{h}$. We take an arbitrary vector $v^{\prime} \in V$, for $\jmath \neq i$. By Lemma (2), $X_{o} \xi \wedge v^{\prime}$ and $X \xi \wedge v_{o}$ are eigenvectors of $\hat{R}$ with distinct eigenvalues $\lambda_{3}$ and $\lambda_{\imath}$, respectively. Therefore they are mutually orthogonal. Hence

$$
\begin{aligned}
0 & =\left\langle X_{o} \xi \wedge v^{\prime}, X \xi \wedge v_{o}\right\rangle \\
& =\left\langle X_{o} \xi, X \xi\right\rangle\left\langle v^{\prime}, v_{o}\right\rangle-\left\langle X_{o} \xi, v_{0}\right\rangle\left\langle X \xi, v^{\prime}\right\rangle \\
& =-\left\langle X_{o} \xi, v_{0}\right\rangle\left\langle X \xi, v^{\prime}\right\rangle .
\end{aligned}
$$

Since $\left\langle X_{0} \xi, v_{0}\right\rangle \neq 0$, we have $\left\langle X \xi, v^{\prime}\right\rangle=0$. From this it follows that $X \xi \in V_{\imath}$. This together with the preceding fact implies for this $i V_{i}+\boldsymbol{R} \cdot \xi$ is an invariant subspace by the action of $\mathfrak{h}$. By the irreducibility of $\mathfrak{h}$, we have $V_{i}+\boldsymbol{R} \cdot \xi=\boldsymbol{R}^{n}$. This means that the whole $V$ is an eigenspace of $\widetilde{R}_{\xi}$, whose eigenvalue will be denoted by $\lambda$. In particular it holds that

$$
R(\xi, x) y=\lambda\{\langle\xi, y\rangle x-\langle x, y\rangle \xi\} \text { for any } x, y \in \boldsymbol{R}^{n} .
$$

Now we define a curvature tensor $R_{0}$ of $\boldsymbol{R}^{n}$ by $R_{0}(x, y) z=\langle y, z\rangle x-\langle x, z\rangle y$ for $x, y, z \in \boldsymbol{R}^{n}$. For the preceding eigenvalue $\lambda$ of $\widetilde{R}_{\xi}$, put a subspace $\mathfrak{R}$ of $\boldsymbol{R}^{n}$ as follows:

$$
\mathfrak{R}=\left\{x \in \boldsymbol{R}^{n} \mid i(x)\left(R+\lambda R_{o}\right)=0\right\} .
$$

Here $i(x)\left(R+\lambda R_{0}\right)$ denotes the $(1,2)$-tensor on $\boldsymbol{R}^{n}$ given by 


$$
i(x)\left(R+\lambda R_{0}\right)(u, v)=\left(R+\lambda R_{0}\right)(x, u) v \text { for } u, v \in \boldsymbol{R}^{n} .
$$

Since $R$ and $R_{o}$ are invariant by the action of $H, \Re$ is an invariant subspace of $\boldsymbol{R}^{n}$ by the action of $H$. By the preceding discussion, we have $\xi \in \Re$. From the irreducibility of $H$, it follows that $\Re=\boldsymbol{R}^{n}$. Therefore it holds that $R(x, y) z=$ $-\lambda\{\langle y, z\rangle x-\langle x, z\rangle y\}$ for $x, y, z \in \boldsymbol{R}^{n}$. Thus our assertion has been proved.

As an application of Theorem 4.8, we obtain the following, which is a slight generalization of Theorem 1 in B. Y. Chen [2].

CoRollary 4.9. Let $M=G / H$ be a Riemannian homogeneous space such that the identity component $H_{o}$ of $H$ acts irreducibly on the tangent space $(\operatorname{dim} M \geqq 3)$. If $M$ admits a totally umbilical hypersurface $S$, then $M$ has constant sectional curvature.

Remark. B. Y. Chen in [2] investigated the case when $M$ is an irreducible Riemannian symmetric space and showed the same conclusion as above. J.A. Wolf ([15]) investigated and classified Riemannian homogeneous spaces which satisfy the assumption stated in Corollary 4.9.

Proof of Corollary 4.9. We can prove this corollary following the same process as the proof of Theorem 1 in [2]. The coset $H$ is denoted by $o$. We can assume that the totally umbilical hypersurface $S$ is through $o . M$ is Einsteinian and hence from the equation of Codazzi, it follows that the mean curvature of $S$ is constant. Using the equation of Codazzi once more, we see that the tangent space $T_{0} S$ is a curvature-invariant hyperplane of $T_{o} M$. The curvature tensor $R$ at $o$ is invariant by the linear isotropy action of $H_{o}$. By Theorem 4.8 we obtain our conclusion.

\section{Curvature-invariant subspaces-consideration of examples}

In this section, we shall classify the curvature-invariant subspaces for curvature tensors of special form. Let $A$ and $B$ be symmetric endomorphisms of $\boldsymbol{R}^{n}$ with respect to the standard inner product $\langle$,$\rangle . We define a (1,3)$-tensor $A \wedge B$ by

$$
A \wedge B(x, y) z=\langle B y, z\rangle A x-\langle B x, z\rangle A y+\langle A y, z\rangle B x-\langle A x, z\rangle B y
$$

(cf. J.P. Bourguignon and H. Karcher [1]). Then $A \wedge B$ is a curvature tensor on $\boldsymbol{R}^{n}$. We try to classify curvature-invariant subspaces with respect to $A \wedge B$. First we fix notations. Given a subspace $V$ in $\boldsymbol{R}^{n}$, we have the orthogonal decomposition $\boldsymbol{R}^{n}=V+V^{\perp}$. For $v \in \boldsymbol{R}^{n}$, we denote by $v_{a}$ and $v_{b}$ the $V$-component and the $V^{\perp}$-component of $v$, respectively. For a symmetric endomorphism $A$ of $\boldsymbol{R}^{n}$, we define a mapping $A^{a}: V \rightarrow V$ and $A^{b}: V \rightarrow V^{\perp}$ by $A^{a} x=(A x)_{a}$ and $A^{b} x$ $=(A x)_{b}$ for $x \in V$, respectively. 
The following is easily shown.

PROPOSITION 5.1. Let $R=A \wedge I$ be a curvature tensor given by a symmetric endomorphism $A$ and an identity transformation $I$ of $\boldsymbol{R}^{n}$ and $V$ be a subspace of $\boldsymbol{R}^{n}$ with $\operatorname{dim} V \geqq 2$. Then $V$ is curvature-invariant with respect to $R$ if and only if $V$ is an invariant subspace of the symmetric endomorphism $A$.

Remark. If a Riemannian manifold $M$ is conformally flat, its curvature tensor $R$ has the form $A \wedge I$ for some symmetric tensor fleld $A$.

Proposition 5.2. Let $R=A \wedge A$ be a curvature tensor given by a symmetric endomorphism $A$ of $\boldsymbol{R}^{n}$ and $V$ be a subspace of $\boldsymbol{R}^{n}$ with $\operatorname{dim} V \geqq 2$. Then $V$ is curvature-invariant with respect to $R$ if and only if one of the following holds:

(1) $V$ is an invariant subspace of the symmetric endomorphism $A$.

(2) $V$ is a null-subspace with respect to $A$, i.e.,

$$
\langle A x, y\rangle=0 \text { for any } x, y \in V
$$

(3) The image $A(V)$ of $V$ by $A$ is of 1-dimensional.

Proof. Let $V$ be a curvature-invariant subspace with respect to $R$. Then we have

$$
\langle A y, z\rangle A^{b} x-\langle A x, z\rangle A^{b} y=0 \text { for any } x, y, z \in V .
$$

We discuss dividing into the following three cases:

Case $1, \operatorname{ker} A^{b}=V$

Case $2, \quad \operatorname{ker} A^{a}=V$

Case $3, \quad \operatorname{ker} A^{a} \neq V$ and $\operatorname{ker} A^{b} \neq V$,

where $\operatorname{ker} A^{a}$ and $\operatorname{ker} A^{b}$ denote the kernel of $A^{a}$ and that of $A^{b}$, respectively.

Case 1 means that $V$ is an invariant subspace of $A$ and Case 2 means that $V$ is a null-subspace with respect to $A$. Therefore we consider Case 3.

ASSERTION 1. $\operatorname{ker} A^{a}=\operatorname{ker} A^{b}$. In particular they coincide with $\operatorname{ker} A \cap V$.

Proof of Assertion 1. First we shall prove ker $A^{b} \subset \operatorname{ker} A^{a}$. We take a non-zero vector $y$ which belongs to the orthogonal complement of $\operatorname{ker} A^{b}$ in $V$. Then $A^{b} y$ is not zero. For $x \in \operatorname{ker} A^{b}$, we have $-\langle A x, z\rangle A^{b} y=0$ and hence $\langle A x, z\rangle=0$ for any $z \in V$. This implies that $x \in \operatorname{ker} A^{a}$.

Next we shall prove $\operatorname{ker} A^{a} \subset \operatorname{ker} A^{b}$. We take a non-zero vector $y$ which belongs to the orthogonal complement of $\operatorname{ker} A^{a}$ in $V$. Then $A^{a} y$ is not zero. For $x \in \operatorname{ker} A^{a}$, we have $\langle A y, z\rangle A^{b} x=0$. Putting $z=A^{a} y$, we obtain $A^{b} x=0$.

We put $V^{\prime}=\operatorname{ker} A^{a}=\operatorname{ker} A^{b}=\operatorname{ker} A \cap V$ and denote by $V^{\prime \prime}$ the orthogonal complement of $V^{\prime}$ in $V$. 
ASSERTION 2. $\operatorname{dim} V^{\prime \prime}=1$.

Proof of Assertion 2. We fix non-zero $x \in V^{\prime \prime}$. Then $A^{a} x$ and $A^{b} x$ are both not zero. For arbitrary $y \in V^{\prime \prime},\left\langle A y, A^{a} x\right\rangle A^{b} x-\left\langle A x, A^{a} x\right\rangle A^{b} y=0$ and hence $A^{b} y=\left(\left\langle A^{a} x, A^{a} y\right\rangle /\left\|A^{a} x\right\|^{2}\right) A^{b} x$. Therefore we have $\operatorname{dim} A^{b}\left(V^{\prime \prime}\right)=1$. Since $A^{b}$ is injective in $V^{\prime \prime}, \operatorname{dim} V^{\prime \prime}=1$.

From these, it follows that in Case $3, \operatorname{dim} A(V)=1$.

Conversely, it is evident that if one of the three conditions in Proposition 5.2 holds for $V$, then $V$ is curvature-invariant with respect to $R=A \wedge A$.

Remark 1. Let $M$ be a hypersurface immersed in a real space form $\tilde{M}(\tilde{c})$ of constant sectional curvature $\tilde{c}$ and $A$ be its shape operator for a unit normal vector field. Then by the equation of Gauss, the curvature tensor $R$ of $M$ has the form $R=1 / 2\{\tilde{c} I \wedge I+A \wedge A\}$. Therefore we can apply Proposition 5.2 to the classification of its curvature-invariant subspaces. In fact, this Proposition will be used in section 6 .

Remark 2. If $A$ is non-singular, the case (3) in Proposition 5.2 does not occur. Moreover if $A$ is positive-definite, the case (1) only may occur.

Proposition 5.3. Let $R=A \wedge B$ be a curvature tensor given by the positivedefinite symmetric endomorphisms $A, B$ of $\boldsymbol{R}^{n}$ and $V$ be a subspace of $\boldsymbol{R}^{n}$ with $\operatorname{dim} V \geqq 3$. Then $V$ is curvature-invariant with respect to $R=A \wedge B$ if and only if one of the following holds:

(1) $V$ is an invariant subspace by both $A$ and $B$.

(2) There exists a positive number $\lambda$ such that

$$
B^{a}=\lambda A^{a} \text { and } B^{b}=-\lambda A^{b} \text { on } V .
$$

(3) There exist a positive number $\lambda$ and a subspace $V^{\prime}$ of codimension 1 . in $V$ such that the following holds:

$$
\begin{aligned}
& \text { (i) } A\left(V^{\prime}\right) \subset V, B\left(V^{\prime}\right) \subset V \text { and } B x=\lambda A x \text { for } x \in V^{\prime} \\
& \text { (ii) } B^{b} y=-\lambda A^{b} y \text { for } y \in V^{\prime \prime} \text {, }
\end{aligned}
$$

where $V^{\prime \prime}$ denotes the orthogonal complement of $V^{\prime}$ in $V$.

Proof. Let $V$ be a curvature-invariant subspace of $\boldsymbol{R}^{n}$ with respect to $R=A \wedge B$. We assume that $\operatorname{dim} V \geqq 3$.

ASSERTION 1. For each $x \in V$, there exists a positive number $\lambda$ (which may depend on $x$ ) such that $B^{b} x=-\lambda A^{b} x$. In particular we have $\operatorname{ker} A^{b}=\operatorname{ker} B^{b}$ and $A^{b}(V)=B^{b}(V)$.

Proof of Assertion 1. Since $\operatorname{dim} V \geqq 3$, there exists a non-zero vector $z \in V$ such that $\langle A x, z\rangle=\langle B x, z\rangle=0$. From (5.1), it follows that $\langle B y, z\rangle A x+$ 
$\langle A y, z\rangle B x \in V$. Hence $\langle B y, z\rangle A^{b} x+\langle A y, z\rangle B^{b} x=0$. We put $y=z$. Since $A$ and $B$ are both positive-definite, $\langle A z, z\rangle>0$ and $\langle B z, z\rangle>0$. Putting $\lambda=$ $\langle B z, z\rangle /\langle A z, z\rangle$, we have $B^{b} x=-\lambda A^{b} x$.

Owing to Assertion 1, we define a subspace $V^{\prime}$ of $V$ by $V^{\prime}=\operatorname{ker} A^{b}=\operatorname{ker} B^{b}$ and denote by $V^{\prime \prime}$ the orthogonal complement of $V^{\prime}$ in $V$.

ASSERTION 2. If $\operatorname{dim} V^{\prime \prime} \geqq 2$, there exists a positive number $\lambda$ such that $B^{a} x=\lambda A^{a} x$ and $B^{b} x=-\lambda A^{b} x$ for any $x \in V$.

Proof of Assertion 2. Let $x$ and $y$ be linearly independent vectors in $V^{\prime \prime}$. By Assertion 1, we have $B^{b} x=-\lambda(x) A^{b} x$ and $B^{b} y=-\lambda(y) A^{b} y$, where $\lambda(x)$ and $\lambda(y)$ denote positive constants depending on $x$ and $y$, respectively. We shall prove that $\lambda(x)=\lambda(y)$. We have

$$
B^{b}(x+y)=-\lambda(x+y) A^{b}(x+y)=-\lambda(x+y) A^{b} x-\lambda(x+y) A^{b} y .
$$

On the other hand,

$$
B^{b}(x+y)=B^{b} x+B^{b} y=-\lambda(x) A^{b} x-\lambda(y) A^{b} y .
$$

Hence

$$
(\lambda(x)-\lambda(x+y)) A^{b} x+(\lambda(y)-\lambda(x+y)) A^{b} y=0 .
$$

Since $A^{b} x$ and $A^{b} y$ are linearly independent, we see that $\lambda(x)=\lambda(x+y)=\lambda(y)$. From this, it follows that the constant $\lambda$ does not depend on the choice of vectors in $V^{\prime \prime}$.

Again, let $x$ and $y$ be linearly independent vectors in $V^{\prime \prime}$. By (5.1), it holds that

$$
\langle B y, z\rangle A^{b} x-\langle B x, z\rangle A^{b} y+\langle A y, z\rangle B^{b} x-\langle A x, z\rangle B^{b} y=0
$$

for any $z \in V$. Hence $\langle B y-\lambda A y, z\rangle A^{b} x-\langle B x-\lambda A x, z\rangle A^{b} y=0$. Since $A^{b} x$ and $A^{b} y$ are linearly independent, we have $\langle B x-\lambda A x, z\rangle=0$. Since $z$ is an arbitrary vector in $V$, we see that $B^{a} x=\lambda A^{a} x$.

Finally, let $x$ and $y$ be a vector in $V^{\prime}$ and a non-zero vector in $V^{\prime \prime}$, respectively. Then we have $-\langle B x, z\rangle A^{b} y-\langle A x, z\rangle B^{b} y=0$ and hence $\langle B x-$ $\lambda A x, z>A^{b} y=0$. Since $A^{b} y \neq 0, B x=\lambda A x$. Therefore it has been shown that there exists a positive number $\lambda$ such that $B^{a} x=\lambda A^{a} x$ and $B^{b} x=-\lambda A^{b} x$ for any $x \in V$.

ASSERTION 3. If $\operatorname{dim} V^{\prime \prime}=1$, there exists a positive number $\lambda$ such that $B x=\lambda A x$ for $x \in V^{\prime}$ and $B^{b} y=-\lambda A^{b} y$ for $y \in V^{\prime \prime}$.

Proof of Assertion 3. From Assertion 1, it follows that $B^{b} y=-\lambda A^{b} y$ for a non-zero vector $y \in V^{\prime \prime}$. Then we have $B x=\lambda A x$ for $x \in V^{\prime}$. It is shown by the same way as Assertion 2. 
The case when $\operatorname{dim} V^{\prime \prime}=0$ means that $A(V) \subset V$ and $B(V) \subset V$. This, together with Assertions 2 and 3 implies that if $V$ is curvature-invariant, one of three conditions in Proposition 5.3 holds.

Conversely, it is easily shown that if one of three conditions in Proposition 5.3 holds for $V, V$ is curvature-invariant with respect to $R=A \wedge B$.

Applying Propositions 5.2 and 5.3, we shall construct examples of curvature tensors which do not all have curvature-invariant subspaces of dimension not less than 3 . We denote by $\left\{e_{1}, \cdots, e_{n}\right\}$ the natural basis of $\boldsymbol{R}^{n}$. Let $A$ be a positive definite symmetric endomorphism of $\boldsymbol{R}^{n}$ such that each $e_{2}(i=1, \cdots, n)$ is an eigenvector of $A$ with mutually distinct eigenvalue $a_{\imath}(\imath=1, \cdots, n)$. For such an $A$, we choose a symmetric endomorphism $B$ which satisfies the following two conditions:

(5.2)-(i) Let $\left(b_{i j}\right)_{i, j=1, \ldots, n}$ be the $n \times n$-matrix which represents $B$ with respect to the natural basis. Then each entry $b_{i}$ is not zero.

(5.2)-(ii) Each eigenspace of $A^{-1} B$ is of dimension 1 .

Here we shall make a remark about the endomorphism $A^{-1} B$ of $\boldsymbol{R}^{n}$. Denote by $g$ a new inner product on $\boldsymbol{R}^{n}$ which is defined by $g(x, y)=\langle A x, y\rangle$ for $x, y \in \boldsymbol{R}^{n}$. Then $A^{-1} B$ is a symmetric endomorphism with respect to this inner product $g$. In fact we have

$$
\begin{aligned}
g\left(A^{-1} B x, y\right) & =\left\langle A A^{-1} B x, y\right\rangle=\langle B x, y\rangle=\langle x, B y\rangle \\
& =\left\langle x, A A^{-1} B y\right\rangle=\left\langle A x, A^{-1} B y\right\rangle=g\left(x, A^{-1} B y\right) .
\end{aligned}
$$

Thus $A^{-1} B$ has real eigenvalues and mutually orthogonal eigenspaces with respect to $g$.

Theorem 5.4. Let $A$ and $B$ be symmetric endomorphisms of $\boldsymbol{R}^{n}$ taken as above. We define a curvature tensor $R_{\varepsilon}$ by $R_{\varepsilon}=A \wedge(A+\varepsilon B)$ for $\varepsilon \in \boldsymbol{R}$. Then for sufficiently small $\varepsilon(\neq 0)$ the curvature tensor $R_{\varepsilon}$ does not at all have curvatureinvariant subspaces of dimension not less than 3.

Proof. We fix an integer $r$ with $3 \leqq r \leqq n-1$. First we shall classify $r$-dimensional curvature-invariant subspaces with respect to $R_{0}=A \wedge A$. By Remark 2 of Proposition 5.2, each $r$-dimensional curvature-invariant subspace with respect to $R_{0}$ is invariant by $A$. Since each eigenspace of $A$ is of dimension 1 , an $r$-dimensional invariant subspace by $A$ is linearly spanned by $e_{\imath_{1}}, \cdots, e_{\imath_{r}}$, $\imath_{1}<\cdots<i_{r}$, where $\left\{e_{1}, \cdots, e_{n}\right\}$ denotes the natural basis of $\boldsymbol{R}^{n}$. Consequently each $r$-dimensional curvature-invariant subspace is given by $\left\{e_{i_{1}}, \cdots, e_{i_{r}}\right\}_{R}$ for some $i_{1}<\cdots<i_{r}$. We note that for sufficiently small $\varepsilon$, curvature-invariant subspaces with respect to $R_{\varepsilon}=A \wedge(A+\varepsilon B)$ lie near those with respect to $R_{0}=$ $A \wedge A$ if they exist. Applying Theorem 4.4, we can easily show this fact. 
Since $A+\varepsilon B$ is a positive definite symmetric endomorphism for sufficiently small $\varepsilon$, we can apply Proposition 5.3 to the curvature tensor $R_{\varepsilon}$. We shall show that if non-zero $\varepsilon$ is sufficiently small, neither of three cases in Proposition 5.3 holds for any $r$-dimensional subspace $V$.

ASSERTION 1. The case (1) in Proposition 5.3 does not hold for any r-dimensional subspace $V$.

Proof of Assertion 1. If $V$ is an invariant subspace by both $A$ and $A+\varepsilon B$, then $V$ is invariant by $B$. An $r$-dimensional invariant subspace $V$ by $A$ is given by $\left\{e_{i_{1}}, \cdots, e_{\imath_{r}}\right\}_{R}$ for some $i_{1}<\cdots<i_{r}$. On the other hand, since $B e_{i_{1}}=$ $\sum_{j=1}^{n} b_{j i_{1}} e_{j}$ and $b_{j i_{1}} \neq 0$ for $j \neq i_{1}, \cdots, i_{r}$ owing to (5.2)-(i), $B e_{\imath_{1}}$ is not contained in $V$. Therefore the case (1) does not hold.

Assertion 2. The case (3) in Proposition 5.3 does not hold for any $r$-dimensional subspace $V$.

Proof of Assertion 2. Suppose that there exist a positive number $\lambda$ and $r$-1-dimensional subspace $V^{\prime}$ in $V$ such that $(A+\varepsilon B) x=\lambda A x$ for any $x \in V^{\prime}$. Accordingly, we have $B x=((\lambda-1) / \varepsilon) A x$ and hence $A^{-1} B x=((\lambda-1) / \varepsilon) x$ for $x \in V^{\prime}$. Therefore $V^{\prime}$ is a subspace of the eigenspace of $A^{-1} B$ with eigenvalue $(\lambda-1) / \varepsilon$. Since $\operatorname{dim} V^{\prime} \geqq 2$, it is contrary to (5.2)-(ii). Therefore the case (3) does not hold.

ASSERTION 3. The case (2) in Proposition 5.3 does not hold for any r-dimensional subspace $V$ if $\varepsilon$ is sufficiently small.

Proof of Assertion 3. First recalling section 4, we prepare some notations. Let $\operatorname{End}\left(F_{r}\right)=F_{r}^{*} \otimes F_{r}$ be the vector bundle consisting of endomorphisms of the natural vector bundle $F_{r}$ over the Grassmann manifold $G_{r}\left(\boldsymbol{R}^{n}\right)$ and $\Gamma \operatorname{End}\left(F_{r}\right)$ be the space of sections of $\operatorname{End}\left(F_{r}\right)$. For $C \in \operatorname{End}\left(\boldsymbol{R}^{n}\right)$ and $V \in G_{r}\left(\boldsymbol{R}^{n}\right)$, we define an endomorphism $\phi(C)_{V}$ of $\left(F_{r}\right)_{V} \cong V$ by restricting $C$ to $V$, i.e.,

$$
\phi(C)_{V}(x)=\text { the } V \text {-component of } C x \text { for } x \in V,
$$

where we take the $V$-component with respect to the orthogonal decomposition $\boldsymbol{R}^{n}=V+V^{\perp}$. Thus we obtain a linear map $\phi: \operatorname{End}\left(\boldsymbol{R}^{n}\right) \rightarrow \Gamma \operatorname{End}\left(F_{r}\right)$. For symmetric endomorphisms $A$ and $B$ in Theorem 5.4 , we define a subset $\mathcal{C}(A ; B)$ of $G_{r}\left(\boldsymbol{R}^{n}\right)$ as follows:

$$
\mathcal{C}(A ; B)=\left\{V \in G_{r}\left(\boldsymbol{R}^{n}\right) \mid \text { there exists a number } \mu \text { such that } \phi(B)_{V}=\mu \phi(A)_{V}\right\} .
$$

It is easily seen that $\mathcal{C}(A ; B)$ is a closed subset in $G_{r}\left(\boldsymbol{R}^{n}\right)$.

Suppose that for $V \in G_{r}\left(\boldsymbol{R}^{n}\right)$, (2) in Proposition 5.3 holds with respect to $R_{\varepsilon}=A \wedge(A+\varepsilon B)$. That is, there exists a positive number $\lambda$ such that $(A+\varepsilon B)^{a}$ $=\lambda A^{a}$ and $(A+\varepsilon B)^{b}=-\lambda A^{b}$ on $V$. Accordingly, it holds that $B^{a}=((\lambda-1) / \varepsilon) A^{a}$ 
on $V$, i.e., $\phi(B)_{V}=((\lambda-1) / \varepsilon) \phi(A)_{V}$. Therefore $V$ belongs to $\mathcal{C}(A ; B)$. On the other hand, we shall show that if $\varepsilon$ is sufficiently small, curvature-invariant subspaces with respect to $R_{\varepsilon}$ must belong to $G_{r}\left(\boldsymbol{R}^{n}\right)-\mathcal{C}(A ; B)$. Let $W \in G_{r}\left(\boldsymbol{R}^{n}\right)$ be a curvature-invariant subspace with respect to $R_{0}=A \wedge A$. Then $W$ does not belong to $\mathcal{C}(A ; B)$. In fact $W$ is given by $\left\{e_{i_{1}}, \cdots, e_{r_{r}}\right\}_{\boldsymbol{R}}$ for some $i_{1}<\cdots<i_{r}$. From our assumptions of $A$ and $B$ it follows that $\phi(A)_{W} e_{\imath_{1}}=a_{\imath_{1}} e_{\imath_{1}}$ and $\phi(B)_{W} e_{\imath_{1}}$ $=b_{i_{1} \imath_{1}} e_{\imath_{1}}+b_{i_{2} \imath_{1}} e_{\imath_{2}}+\cdots+b_{i_{r} \imath_{1}} e_{\imath_{r}}$. Since $b_{i_{2} \imath_{1}} \neq 0$, there does not exist $\mu$ such that $\phi(B)_{W}=\mu \phi(A)_{W}$. We recall that for sufficiently small $\varepsilon$, curvature-invariant subspaces with respect to $R_{\varepsilon}$ must lie near those with respect to $R_{0}$ if they exist. Noticing that $G_{r}\left(\boldsymbol{R}^{n}\right)-\mathcal{C}(A ; B)$ is an open subset in $G_{r}\left(\boldsymbol{R}^{n}\right)$, we obtain our claim.

\section{Totally geodesic submanifolds of hypersurfaces in a Euclidean space}

Let $\tilde{M}^{n+1}(\tilde{c})$ be an $n+1$-dimensional real space form, i. e., a simply connected complete Riemannian manifold with constant sectional curvature $\tilde{c}$. Let $M$ be a hypersurface in $\tilde{M}^{n+1}(\tilde{c})$. Here we assume that $M$ is a regular submanifold of $\tilde{M}^{n+1}(\tilde{c})$. In this section we shall study totally geodesic submanifolds of $M$ applying Proposition 5.2. In particular, we shall classify totally geodesic submanifolds of hypersurfaces in $\boldsymbol{R}^{n+1}$ defined by homogeneous polynomials of degrees 2 and 3.

We fix some notation. We denote $\tilde{\nabla}$ and $\nabla$ the Riemannian connections of $\tilde{M}^{n+1}(\tilde{c})$ and $M$, respectively. We denote by $\xi, \alpha$ and $A$ a unit normal vector field, the second fundamental form, and the shape operator of $M$, respectively.

Proposition 6.1. Let $M$ be a hypersurface in $\tilde{M}^{n+1}(\tilde{c})$ and $S$ be an r-dimensional totally geodesic connected submanifold of $M$. We assume that $\operatorname{dim} A\left(T_{x} S\right)$ $\geqq 2$ at any point $x \in S$. Then one of the following holds:

(1) There exists an $r+1$-dimensional totally geodesic submanifold $\tilde{M}^{r+1}(\tilde{c})$ of $\tilde{M}^{n+1}(\tilde{c})$ which contains $S$ such that an open subset in $\tilde{M}^{r+1}(\tilde{c}) \cap M$ containing $S$ is an $r$-dimensional regular submanifold of $M$ and $S$ is its open submanifold.

(2) There exists an r-dimensional totally geodesic submanifold $\tilde{M}^{r}(\tilde{c})$ of $\tilde{M}^{n+1}(\tilde{c})$ in which $S$ is an open submanifold.

Proof. From the equation of Gauss, it follows that at $x \in M$ the curvature tensor $R$ of $M$ is given by

$$
R(X, Y) Z=\tilde{c}\{\langle Y, Z\rangle X-\langle X, Z\rangle Y\}+\{\langle A Y, Z\rangle A X-\langle A X, Z\rangle A Y\}
$$

$X, Y, Z \in T_{x} M$. Since $S$ is a totally geodesic submanifold of $M$, the tangent space $T_{x} S$ is a curvature-invariant subspace of $T_{x} M$ at any point $x \in S$. Since $\operatorname{dim} A\left(T_{x} S\right) \geqq 2$, by Proposition 5.2 one of the following holds for $T_{x} S$ :

(1) $T_{x} S$ is invariant by $A$, i.e., $A\left(T_{x} S\right) \subset T_{x} S$.

(2) $T_{x} S$ is a null-subspace with respect to $A$, i.e., $\langle A X, Y\rangle=0$ for any $X, Y \in T_{x} S$. 
We define subsets $S_{1}$ and $S_{2}$ in $S$ as follows:

$$
\begin{aligned}
& S_{1}=\left\{x \in S \mid T_{x} S \text { is invariant by } A\right\} \\
& S_{2}=\left\{x \in S \mid T_{x} S \text { is a null-subspace with respect to } A\right\} .
\end{aligned}
$$

Then we have $S=S_{1} \cup S_{2}$ and $S_{1} \cap S_{2}=\emptyset$ (empty). Clearly, $S_{1}$ and $S_{2}$ are both closed subsets in $S$ and hence open. Since $S$ is connected, we have $S=S_{1}$ or $S=S_{2}$.

Let $\left.T \tilde{M}\right|_{S}$ be the vector bundle over $S$ induced by the tangent bundle $T \tilde{M}$ of $\tilde{M}$. Then we have the orthogonal decomposition:

$$
\left.T \tilde{M}\right|_{S}=T S+T^{\perp} S+\boldsymbol{R} \cdot \xi,
$$

where $T^{\perp} S$ denotes the orthogonal complement of $T S$ in $\left.T M\right|_{s}$. We have the following formulas: for $X, Y \in \Gamma T S$

$$
\begin{aligned}
& \tilde{\nabla}_{X} Y=\nabla_{X} Y+\langle\alpha(X, Y), \xi\rangle \xi \\
& \tilde{\nabla}_{X} \xi=-A X .
\end{aligned}
$$

We note that $\nabla_{X} Y \in \Gamma T S$, since $S$ is a totally geodesic submanifold of $M$.

We consider the case $S=S_{1}$, TS is an invariant subbundle of $T M$ by $A$. This, together with (6.2), implies that $T S+\boldsymbol{R} \cdot \xi$ is a parallel subbundle of $\left.T \tilde{M}\right|_{S}$ with respect to $\tilde{\nabla}$. Therefore by the well-known reduction theorem (J. Erbacher [6]), it follows that there exists an $r+1$-dimensional totally geodesic submanifold $\tilde{M}^{r+1}(\tilde{c})$ of $\tilde{M}^{n+1}(\tilde{c})$ such that $S$ is a submanifold of $\tilde{M}^{r+1}(\tilde{c})$. In particular, we have $S \subset \tilde{M}^{r+1}(\tilde{c}) \cap M$. Since $T_{x} \tilde{M}^{r+1}=T_{x} S+\boldsymbol{R} \cdot \xi_{x}$ at an arbitrary point $x \in S, \tilde{M}^{r+1} \cap M$ is an $r$-dimensional regular submanifold of $\tilde{M}^{r+1}$ in a neighborhood of $S$ and $S$ is its open submanifold. Since both $M$ and $\tilde{M}^{r+1}(\tilde{c})$ are regular submanifolds of $\tilde{M}^{n+1}(\tilde{c})$, an open subset in $\tilde{M}^{r+1} \cap M$ containing $S$ is also a regular submanifold of $M$.

If $S=S_{2}$, by (6.1), $S$ is a totally geodesic submanifold of $\tilde{M}^{n+1}(\tilde{c})$. Hence we obtain our assertion (2) in Proposition 6.1.

Now we shall consider the converse of Proposition 6.1. Let $S$ be an $r$-dimensional submanifold of $M$. If $S$ is a totally geodesic submanifold of $\tilde{M}^{n+1}(\tilde{c})$, evidently it is a totally geodesic submanifold of $M$. Corresponding to Proposition 6.1 (1), the following is easily seen.

LEMMA 6.2. Let $\tilde{M}^{r+1}(\tilde{c})$ be an $r+1$-dimensional totally geodesic submanifold of $\tilde{M}^{n+1}(\tilde{c})$. Suppose that $S=\tilde{M}^{r+1} \cap M$ is an r-dimensional submanifold of $\tilde{M}^{n+1}(\tilde{c})$ (and hence it is also a submanifold of both $\tilde{M}^{r+1}$ and $M$ ). Let $\xi$ be a unit normal vector field of $M$. If $\xi_{x} \in T_{x} \tilde{M}^{r+1}$ at an arbitrary point $x \in S$, then $S$ is a totally geodesic submanifold of $M$. 
Let $\boldsymbol{R}^{n+1}$ be an $n+1$-dimensional real vector space with standard inner product $\langle$,$\rangle . Let F$ be a symmetric multilinear form of degree $m$ on $\boldsymbol{R}^{n+1}$ and $f$ be a homogeneous polynomial defined by $f(x)=F(x, \cdots, x)$ for $x \in \boldsymbol{R}^{n+1}$. We set a subset $M$ of $\boldsymbol{R}^{n+1}$ by $M=\left\{x \in \boldsymbol{R}^{n+1} \mid f(x)=1\right\}$. As usual, we identify the tangent space $T_{x} \boldsymbol{R}^{n+1}$ of $\boldsymbol{R}^{n+1}$ at $x$ with $\boldsymbol{R}^{n+1}$. Under this identification, we have $d f_{x}(v)=m F(x, \cdots, x, v)$ for $v \in T_{x} \boldsymbol{R}^{n+1} \cong \boldsymbol{R}^{n+1}$. Since $d f_{x}(x)=m \neq 0$ at $x \in M$, $M$ is a smooth hypersurface of $\boldsymbol{R}^{n+1}$ if $M$ is not empty. Easily we see that $M$ is a real analytic and complete Riemannian manifold. We try to classify totally geodesic submanifolds of such hypersurfaces.

We consider two types of totally geodesic submanifolds:

Type I. We take an $r+1$-dimensional linear subspace $W$ of $\boldsymbol{R}^{n+1}$ and a vector $C \in W^{\perp}$, where $W^{\perp}$ denotes the orthogonal complement of $W$ in $\boldsymbol{R}^{n+1}$. We denote by $C+W$ the affine subspace defined by $C+w, w \in W$. Let $S$ be one of connected components of $(C+W) \cap M$. Now we assume that

$$
F\left(x, \cdots, x, W^{\perp}\right)=0 \text { at any point } x \in S .
$$

Type II. We take a point $x \in M$ and an $r$-dimensional linear subspace $W$. Now we assume that

$$
F(\overbrace{x, \cdots, x}^{k}, \overbrace{W, \cdots, W}^{m-k})=0 \text { for } 0 \leqq k \leqq m-1 .
$$

Proposition 6.3. (1) Let $S$ be a subset of $M$ given in Type I above. Then $S$ is an $r$-dimensional totally geodesic submanifold of $M$.

(2) Let $x$ and $W$ be a point of $M$ and an $r$-dimensional subspace given in Type II, respectively. Then the affine subspace $x+W$ is contained in $M$ and hence it is an $r$-dimensional totally geodesic submanifold of $M$.

Totally geodesic submanifolds in both cases are maximal in the sense of Theorem 3.5.

Proof. (1) First we shall show that $S$ is a smooth hypersurface in an $r+1$-dimensional affine subspace $C+W$. Let $x$ be an arbitrary point of $S$. We can identify the tangent space $T_{x}(C+W)$ with $W$. If $d f_{x}(w)=m F(x, \cdots, x, w)$ $=0$ for all $w \in T_{x}(C+W) \cong W$, then the condition (6.3) implies that $F(x, \cdots x, v)$ $=0$ for all $v \in \boldsymbol{R}^{n+1}$. It contradicts to $F(x, \cdots, x)=1$. Therefore there exists a vector $w \in T_{x}(C+W) \cong W$ such that $d f_{x}(w) \neq 0$. Hence $S$ is a smooth hypersurface in $C+W$.

We note that the tangent space $T_{x} M$ is given by

$$
T_{x} M=\left\{v \in T_{x} \boldsymbol{R}^{n+1} \cong \boldsymbol{R}^{n+1} \mid d f_{x}(v)=m F(x, \cdots, x, v)=0\right\} .
$$

From (6.3), it follows that $W^{\perp} \subset T_{x} M$ at every point $x \in S$. Let $\xi$ be the unit normal vector field of $M$ in $\boldsymbol{R}^{n+1}$. Then at every point $x \in S, \boldsymbol{\xi}_{x}$ is orthogonal to $W^{\perp}$. Consequently we have $\xi_{x} \in W \cong T_{x}(C+W)$. By Lemma 6.2, $S$ is a totally geodesic submanifold of $M$. 
(2) For an arbitrary vector $w \in W$, the condition (6.4) implies

$$
F(x+w, \cdots, x+w)=\sum_{k=0}^{m-1}\left(\begin{array}{c}
m \\
k
\end{array}\right) F(\overbrace{x, \cdots, x}^{k}, \overbrace{w, \cdots, w}^{m-k})+F(x, \cdots, x)=1 .
$$

Therefore the affine subspace $x+W$ is contained in $M$.

Since totally geodesic submanifolds given above are complete Riemannian manifold and imbedded in $M$, they are maximal in the sense of Theorem 3.5.

Remark. Let $\phi$ be a linear transformation of $\boldsymbol{R}^{n+1}$. We define a multilinear form $F^{\prime}$ by $F^{\prime}=\phi \cdot F$, i.e., $F^{\prime}\left(v_{1}, \cdots, v_{m}\right)=F\left(\phi^{-1} v_{1}, \cdots, \phi^{-1} v_{m}\right)$ and denote by $M^{\prime}$ the hypersurface of $\boldsymbol{R}^{n+1}$ defined by $F^{\prime}$. Then we have $\phi(M)=M^{\prime}$. Moreover if an affine subspace $x+W$ is contained in $M$, then the affine subspace $\phi(x+W)$ is contained in $M^{\prime}$ and vice versa. The existence of totally geodesic submanifolds of Type II is a problem which belongs to affine differential geometry.

We add one more assumption to $F$. We say that a symmetric multilinear form $F$ is non-singular when $F(x, \cdots, x, v)=0$ for $v \in \boldsymbol{R}^{n+1}$ only if $x=0$.

THEOREM 6.4. Let $F$ be a non-singular symmetric multilinear form of degree $m$ on $\boldsymbol{R}^{n+1}$ and $M$ be a hypersurface defined by $F$. Let $S$ be an r-dimensional $(2 \leqq r \leqq n-1)$ connected Riemannian manifold and $\varphi$ be a totally geodesic immersion of $S$ into $M$. Then there exists an $r$-dimensional totally geodesic submanifold $\widetilde{S}$ obtained by Proposition 6.3 such that $\varphi(S) \subset \widetilde{S}$. That is, maximal totally geodesic submanifolds are exhausted by ones given in Proposition 6.3. In particular, a maximal totally geodesic submanifold of $M$ is imbedded and closed in $M$.

Proof. We note that $S$ is a real analytic Riemannian manifold and $\varphi$ is a real analytic immersion. Let $\varphi^{-1} T M$ be an induced bundle by $\varphi$ from the tangent bundle of $M$. Then we have an orthogonal decomposition: $\varphi^{-1} T M=$ $T S+T^{\perp} S$. We denote by $A$ the shape operator of $M$ in $\boldsymbol{R}^{n+1}$. We view it as a section of the endomorphism bundle $\operatorname{End}\left(\varphi^{-1} T M\right)$. We put $k=\max _{x \in S} \operatorname{dim} A\left(T_{x} S\right)$. Then the following three cases may occur:

Case 1. $k=0$.

Case 2. $k=1$.

Case 3. $k \geqq 2$.

ASSERTION 1. Case 1 does not occur.

Proof of Assertion 1. Since our discussion below is local, we don't distinguish $S$ and $\varphi(S)$ and may assume that $S$ is an imbedded submanifold of $M$. We shall show that at a fixed point $x \in S$, the following holds:

$$
F(u, \cdots, u, v)=0 \text { for any } u \in T_{x} S \text { and } v \in \boldsymbol{R}^{n+1} .
$$

Therefore it contradicts that $F$ is non-singular. Now we shall prove (6.5). In our case $S$ is a totally geodesic submanifold of $\boldsymbol{R}^{n+1}$. For $x \in S$ and $u \in T_{x} S$, 
we put $\gamma(t)=x+t u$. Then there exists a positive number $\varepsilon$ such that $\gamma(t) \in S$ for $|t|<\varepsilon$. Since $A(T S)=0$ on $S$, the tangent bundle $T M$ is a parallel subbundle of $T \boldsymbol{R}^{n+1}$ along $\gamma$ with respect to $\tilde{\nabla}$. Under a usual identification, we see that if $v \in T_{x} M$, then $v \in T_{\gamma(t)} M$ for $|t|<\varepsilon$. Therefore we have

$$
\begin{aligned}
0 & =\frac{1}{m} d f_{\gamma(t)}(v)=F(\gamma(t), \cdots, \gamma(t), v) \\
& =F(x, \cdots, x, v)+(m-1) t F(x, \cdots, x, u, v)+\cdots+t^{m-1} F(u, \cdots, u, v) .
\end{aligned}
$$

Hence it follows that $F(u, \cdots, u, v)=0$ for any $v \in T_{x} M$. Since $\gamma(t) \in S \subset M$ for $|t|<\varepsilon$, we have

$$
\begin{aligned}
1 & =F(\gamma(t), \cdots, \gamma(t)) \\
& =1+m t F(x, \cdots, x, u)+\cdots+m t^{m-1} F(u, \cdots, u, x)+t^{m} F(u, \cdots, u) .
\end{aligned}
$$

Hence it follows that $F(u, \cdots, u, x)=0$. Consequently we obtain the formula (6.5).

ASSERTION 2. In Case 2 at any point $x \in S, T_{x} S$ is a null-subspace with respect to $A$ i.e., $\langle A X, Y\rangle=0$ for any $X, Y \in T_{x} S$. Therefore $S$ is a totally geodesic submanifold of $\boldsymbol{R}^{n+1}$.

Proof of Assertion 2. Suppose that at a point $x \in S, T_{x} S$ is not a nullsubspace with respect to $A$. Then there exists a neighborhood $S^{\prime}$ of $x$ in $S$ such that at each $y \in S^{\prime}, T_{y} S^{\prime}$ is not a null-subspace with respect to $A$. We define a subspace $\mathfrak{D}_{y}$ of $T_{y} S^{\prime}$ at $y \in S^{\prime}$ by $\mathscr{D}_{y}=\left\{X \in T_{y} S^{\prime} \mid A X=0\right\}$. Then $\mathscr{D}$ is a distribution in $S^{\prime}$ with codimension 1 . $\mathfrak{D}$ is exactly a relative nullity distribution when we view $S^{\prime}$ as a submanifold of $\boldsymbol{R}^{n+1}$. Therefore $\mathscr{D}$ is involutive and each leaf of $\mathfrak{D}$ is a totally geodesic submanifold of $S^{\prime}$. We denote by $N$ one of leaves of $\mathfrak{D}$. Then $N$ is a totally geodesic submanifold of $M$ which satisfies $A\left(T_{y} N\right)=0$ at any point $y \in N$. By the proof of Assertion 1, we see that Assertion 1 is also true when $S$ is a 1 -dimensional totally geodesic submanifold of $M$, i.e., a geodesic. Therefore such a submanifold $N$ does not exist. It's a contradiction. Hence our assertion has been proved.

Now we consider Case 3. There exists an open subset $S^{\prime}$ in $S$ such that $\operatorname{dim} A\left(T_{x} S\right) \geqq 2$ at each point $x \in S^{\prime}$. If there exists an $r$-dimensional totally geodesic submanifold $\widetilde{S}$ obtained by Proposition 6.3 such that $\varphi\left(S^{\prime}\right) \subset \widetilde{S}$, then by the real analyticity of $\varphi$ it holds that $\varphi(S) \subset \widetilde{S}$. Therefore we may discuss, assuming that $S$ is an imbedded submanifold of $M$ and $\operatorname{dim} A\left(T_{x} S\right) \geqq 2$ at each point $x \in S$. By Proposition 6.1, one of the following holds :

Case $3-i$. There exists an $r+1$-dimensional affine subspace $\tilde{M}^{r+1}$ which contains $S$ such that an open subset in $\tilde{M}^{r+1} \cap M$ containing $S$ is an $r$-dimensional regular submanifold of $M$ and $S$ is its open submanifold. 
Case 3-ii. There exists an $r$-dimensional affine subspace $\tilde{M}^{r}$ in which $S$ is an open submanifold.

Now we consider Case 3-i. We note that by the proof of Proposition 6.1 $\xi_{x} \in T_{x} \tilde{M}^{r+1}$ at any point $x \in S$. Let $\widetilde{S}$ be a connected component of $\tilde{M}^{r+1} \cap M$ which contains $S$.

ASSERTION 3. $\tilde{S}$ is an $r$-dimensional regular submanifold of $M$ and moreover $\tilde{S}$ is a complete totally geodesic submanifold of $M$.

Proof of Assertion 3. We fix a point $p \in S$. Applying Corollary 3.7 to $T_{p} S$, we see that there is a complete totally geodesic submanifold $\bar{S}$ immersed in $M$ which contains $S$ as an open submanifold. We denote by $\phi$ the totally geodesic immersion of $\bar{S}$ into $M$. By the real analyticity of $\phi$, we have $\phi(\bar{S}) \subset \tilde{M}^{r+1}$ and hence $\phi(\bar{S}) \subset \widetilde{S}$. In the same manner, by the real analyticity of $\xi$, it holds that $\xi_{\phi(q)} \in T_{\phi(q)} \tilde{M}^{r+1}$ at any point $q \in \bar{S}$. We put $\bar{S}=\phi(\bar{S})$. We shall prove that $\bar{S}$ is an $r$-dimensional regular submanifold of $M$. We denote by $\tilde{f}$ the polynomial function on $\tilde{M}^{r+1}$ obtained by restricting $f(x)=F(x, \cdots, x)$ to $\tilde{M}^{r+1}$. Then we have $\tilde{M}^{r+1} \cap M=\left\{x \in \tilde{M}^{r+1} \mid \tilde{f}(x)=1\right\}$. Since $\xi_{\phi(q)} \in T_{\phi(q)} \tilde{M}^{r+1}$ at an arbitrary point $q \in \bar{S}, d \tilde{f}_{\phi(q)} \neq 0$ in the cotangent space $T_{\phi(q)}^{*} \tilde{M}^{r+1}$. Therefore there exists a neighborhood $U$ of $\phi(q)$ in $\tilde{M}^{r+1}$ such that $M \cap U=$ $\{x \in U \mid \tilde{f}(x)=1\}$ is an $r$-dimensional regular submanifold of $\tilde{M}^{r+1}$. Hence we can take a neighborhood $\bar{U}$ of $q$ in $\bar{S}$ such that $\phi$ is a diffeomorphism of $\bar{U}$ onto $M \cap V$, where $V$ is an adequate neighborhood of $\phi(q)$ in $\tilde{M}^{r+1}$. From this, it follows that $\overline{\bar{S}} \cap V=M \cap V$. Consequently it has been proved that $\bar{S}$ is an $r$-dimensional regular submanifold of $\tilde{M}^{r+1}$ and hence is also a regular submanifold of $M$. Moreover $\phi$ is a Riemannian covering map of $\bar{S}$ onto $\bar{S}$. Since $\bar{S}$ is a complete Riemannian manifold, so is $\overline{\bar{S}}$. In our proof above, it has been also shown that $\overline{\bar{S}}$ is an open subset of $\widetilde{S}$.

Finally we shall show that $\bar{S}$ is a closed subset of $\tilde{S}$. If this is shown, we have $\overline{\bar{S}}=\widetilde{S}$ and obtain our assertion. Let $\left\{p_{j}\right\}$ and $p$ be a sequence of points in $\overline{\bar{S}}$ and a point of $\widetilde{S}$ which satisfy $\lim _{\sigma^{\rightarrow \infty}} p_{\jmath}=p$ in $\widetilde{S}$. Since $\xi_{p_{j}} \in T_{p_{j}} \tilde{M}^{r+1}$ for any $j$, then we have $\xi_{p} \in T_{p} \tilde{M}^{r+1}$. Therefore there exists a neighborhood $V$ of $p$ in $\tilde{M}^{r+1}$ such that $M \cap V$ is an $r$-dimensional regular submanifold of $\tilde{M}^{r+1}$. We choose $p$, which belongs to a normal coordinate neighborhood $Q$ of $p$ in $M \cap V$. Let $\gamma$ be a geodesic from $p$, to $p$ in $C$. We parametrize $\gamma$ such that $\gamma(0)=p$, and $\gamma(1)=p$. Since $\overline{\bar{S}} \cap V$ is an open submanifold of $M \cap V$, there exists an open interval $I \subset[0,1]$ containing 0 such that $\gamma(I)$ is contained in $\bar{S}$ and $\left.\gamma\right|_{I}$ is a geodesic of $\overline{\bar{S}}$. Since $\overline{\bar{S}}$ is a complete Riemannian manifold, we have $I=[0,1]$ and hence $p=\gamma(1)$ belongs to $\bar{S}$. Consequently it has been proved that $\overline{\bar{S}}$ is a closed subset of $\tilde{S}$.

We continue our discussion on Case $3-\mathrm{i}$. We denote by $W$ an $r+1$-dimensional linear subspace of $\boldsymbol{R}^{n+1}$ which corresponds to the tangent space $T_{x} \tilde{M}^{r+1}$ at $x \in \tilde{M}^{r+1}$ under the usual identification $T_{x} \boldsymbol{R}^{n+1} \cong \boldsymbol{R}^{n+1}$ and denote by $W^{\perp}$ the 
orthogonal complement of $W$ in $\boldsymbol{R}^{n+1}$. Let $C$ be a vector of $W^{\perp}$ which is the image of $\tilde{M}^{r+1}$ by the orthogonal projection of $\boldsymbol{R}^{n+1}$ onto $W^{\perp}$. Then the affine subspace $\tilde{M}^{r+1}$ is given by $C+W$. As has been shown in Assertion 3, for an arbitrary point $x \in \tilde{S}, \xi_{x}$ belongs to $T_{x} \tilde{M}^{r+1}$ and is orthogonal to $W^{\perp}$. Therefore we have $F\left(x, \cdots, x, W^{\perp}\right)=(1 / m) d f_{x}\left(W^{\perp}\right)=0$ and obtain the formula (6.3).

Finally we consider Case 2 and Case 3 -ii. In each case, there exists an $r$-dimensional affine subspace $\tilde{M}^{r}$ in which $S$ is an open submanifold. We denote by $W$ an $r$-dimensional linear subspace of $\boldsymbol{R}^{n+1}$ which corresponds to $T_{x} S=T_{x} \tilde{M}^{r}$. For an arbitrary vector $w \in W$, we take a straight line $\gamma(t)=x+t w$. For sufficiently small $t, \gamma(t)$ belong to $S$ and hence to $M$. Therefore we have

$$
\begin{aligned}
1 & =F(\gamma(t), \cdots, \gamma(t)) \\
& =F(x, \cdots, x)+\cdots+\left(\begin{array}{c}
m \\
k
\end{array}\right) t^{m-k} F(\overbrace{x, \cdots, x}^{k}, \overbrace{w, \cdots, w}^{m-k})+\cdots t^{m} F(w, \cdots, w) .
\end{aligned}
$$

Hence we have $F(\overbrace{x, \cdots, x}^{k}, \overbrace{w, \cdots, w}^{m-k})=0$ for $0 \leqq k \leqq m-1$. Thus $\gamma(t)$ for all $t \in \boldsymbol{R}$ belong to $M$ and hence the affine subspace $x+W=\tilde{M}^{r}$ is contained in $M$.

In the rest of this section we shall classify totally geodesic submanifolds of hypersurfaces defined by symmetric bilinear forms and trilinear forms on $\boldsymbol{R}^{n+1}$. Let $F$ be a non-singular symmetric bilinear form on $\boldsymbol{R}^{n+1}$. We use the same symbol $F$ for the symmetric endomorphism of $\boldsymbol{R}^{n+1}$ which corresponds to the symmetric bilinear form by the standard inner product $\langle$,$\rangle . Let M$ be a hypersurface defined by $F$. Then the following holds.

THEOREM 6.5. If $S$ is an $r$-dimensional $(2 \leqq r \leqq n-1)$ maximal totally geodesic submanifold of $M$, then $S$ is one of the following:

(1) a connected component of $W \cap M$, where $W$ is an $r+1$-dimensional invariant subspace by $F$,

(2) an r-dimensional affine subspace of $\boldsymbol{R}^{n+1}$ which is contained in $M$.

Proof. It is sufficient to consider Case 3-i in the proof of Theorem 6.4. Namely there exist an $r+1$-dimensional linear subspace $W$ and a vector $C \in W^{\perp}$ and $S$ is a connected component of $(C+W) \cap M$ which satisfies $F(x, v)=0$ for any $x \in S$ and any $v \in W^{\perp}$. Let $v$ be an arbitrary vector of $W^{\perp}$. We view $F$ and $v$ as parallel tensor fields with respect to the Riemannian connection $\tilde{\nabla}$ of $\boldsymbol{R}^{n+1}$. For vector fields $U_{1}$ and $U_{2}$ over $S$, we have

and

$$
\begin{aligned}
0 & =\left(\tilde{\nabla}_{U_{1}} F\right)(x, v)=U_{1} F(x, v)-F\left(U_{1}, v\right) \\
& =-F\left(U_{1}, v\right)
\end{aligned}
$$

$$
\begin{aligned}
0 & =\left(\tilde{\nabla}_{U_{2}} F\right)\left(U_{1}, v\right)=U_{2} F\left(U_{1}, v\right)-F\left(\tilde{\nabla}_{U_{2}} U_{1}, v\right) \\
& =-F\left(\alpha\left(U_{2}, U_{1}\right), v\right) .
\end{aligned}
$$


From the above computations, it follows that at point $x \in S$

$$
\begin{aligned}
& F(x, v)=0, \\
& F(u, v)=0 \text { for any } u \in T_{x} S, \\
& F\left(\alpha\left(u_{1}, u_{2}\right), v\right)=0 \text { for any } u_{1}, u_{2} \in T_{x} S .
\end{aligned}
$$

We may assume that $\alpha$ restricted to $T_{x} S$ is not zero. Therefore there are $u_{1}$ and $u_{2}$ in $T_{x} S$ such that $\alpha\left(u_{1}, u_{2}\right) \neq 0$. The above last equation implies that $F\left(\xi_{x}, v\right)=0$. Since $W=T_{x} S+\boldsymbol{R} \xi_{x}$, we obtain $F\left(W, W^{\perp}\right)=0$. Hence $W$ is an invariant subspace by $F$. Since $F\left(x, W^{\perp}\right)=0$, we have $F\left(C, W^{\perp}\right)=0$. On the other hand, since $C \in W^{\perp}$, we have $F(C, W)=0$. From the non-singularity of $F$, it follows that $C=0$. Consequently $S$ is a connected component of $W \cap M$.

By fundamental properties of symmetric bilinear forms we can show the following.

Proposition 6.6. In Theorem 6.5, we assume that $F$ has the index $\nu$. Then the dimension of an affine subspace contained in $M$ is not greater than the minimum of $\nu$ and $n-\nu$. Let $\widetilde{S}_{1}$ and $\widetilde{S}_{2}$ be affine subspaces of the same dimension which are contained in $M$. Then there exists a linear transformation $\phi$ of $\boldsymbol{R}^{n+1}$ which preserves $F$ such that $\phi\left(\widetilde{S}_{1}\right)=\widetilde{S}_{2}$.

Examples. Let $F$ be a symmetric bilinear form of $\boldsymbol{R}^{n+1}$ given by $F=$ $\sum_{l=1}^{n+1} a_{i} e_{i}^{*} \otimes e_{i}^{*}$, where $\left\{e_{1}^{*}, \cdots, e_{n+1}^{*}\right\}$ denotes the dual basis of the natural basis $\left\{e_{1}, \cdots, e_{n+1}\right\}$ of $\boldsymbol{R}^{n+1}$. If every $a_{\imath}$ is positive, the hypersurface $M$ defined by $F$ does not contain any affine subspace of positive dimension. In addition suppose that $a_{1}, \cdots, a_{n+1}$ are mutually distinct. Then the number of $F$-invariant $r+1$-dimensional subspaces is $\left(\begin{array}{l}n+1 \\ r+1\end{array}\right)$. We put $M^{\prime}=\left\{x={ }^{t}\left(x^{1}, \cdots, x^{n+1}\right) \in M \mid x^{2} \neq 0\right.$ for any $i$. Then $M^{\prime}$ is an open submanifold of $M$. The Riemannian manifold $M^{\prime}$ does not have any $r(2 \leqq r \leqq n-1)$-dimensional totally geodesic submanifolds. In fact, the $F$-invariant subspace which contains a point $x \in M^{\prime}$ is the whole space $\boldsymbol{R}^{n+1}$.

Next we consider the following symmetric trilinear form $F$ on $\boldsymbol{R}^{n+1}$ : $F=\sum_{\imath=1}^{n+1} e_{i}^{*} \otimes e_{i}^{*} \otimes e_{i}^{*}$. Then $F$ is non-singular in our sense. In the rest of this section, we denote by $Q^{n}$ the hypersurface of $\boldsymbol{R}^{n+1}$ defined by $F$, i.e.,

$$
\begin{aligned}
Q^{n} & =\left\{x \in \boldsymbol{R}^{n+1} \mid F(x, x, x)=1\right\} \\
& =\left\{x={ }^{t}\left(x^{1}, \cdots, x^{n+1}\right) \in \boldsymbol{R}^{n+1} \mid \sum_{\imath=1}^{n+1}\left(x^{i}\right)^{3}=1\right\} .
\end{aligned}
$$

Let $S_{n+1}$ be the symmetric group consisting of permutations of $\{1,2, \cdots, n+1\}$. For $\sigma \in S_{n+1}$, we define a linear transformation $T_{\sigma}$ of $\boldsymbol{R}^{n+1}$ as follows: $T_{\sigma}\left(e_{2}\right)=$ $e_{\sigma(i)}(i=1, \cdots, n+1)$. Then $T_{\sigma}$ is an orthogonal transformation which preserves 
the above trilinear form $F$. Hence $T_{\sigma}$ yields an isometry of Riemannian manifold $Q^{n}$.

We shall classify totally geodesic submanifolds of $Q^{n}$. First we present examples of totally geodesic submanifolds. For $2 \leqq m \leqq n-1, Q^{m}$ can be viewed as a submanifold of $Q^{n}$, naturally, i.e.,

$$
Q^{m}=\left\{x={ }^{t}\left(x^{1}, \cdots, x^{n+1}\right) \in Q^{n} \mid x^{m+2}=\cdots=x^{n+1}=0\right\} .
$$

Let $W$ be an $m+1$-dimensional linear subspace of $\boldsymbol{R}^{n+1}$ spanned by $e_{1}, \cdots, e_{m+1}$. Its orthogonal complement $W^{\perp}$ is spanned by $e_{m+2}, \cdots, e_{n+1}$. Then evidently it holds that $Q^{m}=W \cap Q^{n}$ and $F(x, y, v)=0$ for $x, y \in W, v \in W^{\perp}$. By Proposition 6.3 (1), $Q^{m}$ is totally geodesic in $Q^{n}$. Another example is given as follows: Let $k_{1}, \cdots, k_{l}$ be integers which satisfy $2 \leqq k_{1} \leqq \cdots \leqq k_{l}$ and $k_{1}+\cdots+k_{l} \leqq n+1$. We put

$$
\begin{aligned}
& S=\left\{x={ }^{t}\left(x^{1}, \cdots, x^{n+1}\right) \in Q^{n} \mid x^{1}=\cdots=x^{k_{1}}, x^{k_{1}+1}=\cdots=x^{k_{1}+k_{2}},\right. \\
& \left.\cdots, x^{k_{1}+\cdots+k_{l-1}+1}=\cdots=x^{k_{1}+\cdots+k} l-1+k l\right\} .
\end{aligned}
$$

Then it is easily seen that $S$ is connected and is a submanifold of $Q^{n}$ with codimension $k_{1}+\cdots+k_{l}-l$. We define cyclic permutations $\sigma_{1}, \cdots, \sigma_{l}$ by $\sigma_{1}=$ $\left(1, \cdots, k_{1}\right), \cdots, \sigma_{l}=\left(k_{1}+\cdots+k_{l-1}+1, \cdots, k_{1}+\cdots+k_{l-1}+k_{l}\right)$ and a permutation $\sigma$ by $\sigma=\sigma_{1} \cdots \sigma_{l}$. Then $S$ is a fixed point set of $Q^{n}$ by $T_{\sigma}$. Therefore $S$ is totally geodesic in $Q^{n}$. For convenience, we denote this submanifold by $S_{k_{1}, \cdots, k_{l}}$ in this section. We shall show that these submanifolds and affine subspaces exhaust totally geodesic submanifolds of $Q^{n}$.

THEOREM 6.7. If $S$ is an $r$-dimensional maximal totally geodesic submanifold of $Q^{n}$, then $S$ is congruent to one of the following totally geodesic submanifolds by an isometry $T_{\sigma}, \sigma \in S_{n+1}$ :

(1) $S_{k_{1}, \cdots, k_{l}}$ in $Q^{m}$ for some $m \leqq n$, where $r=m+l-\left(k_{1}+\cdots+k_{l}\right)$,

(2) an $r$-dimensional affine subspace of $\boldsymbol{R}^{n+1}$ which is contained in $Q^{n}$.

Proof. Owing to Theorem 6.4, we consider Case 3-i in the proof of Theorem 6.4. Namely, there exist an $r+1$-dimensional linear subspace $W$ and a vector $C \in W^{\perp}$ and $S$ is a connected component of $(C+W) \cap Q^{n}$ which satisfies $F(x, x, v)=0$ for any $x \in S$ and $v \in W^{\perp}$. Let $\xi, A, \alpha$, and $\nabla \alpha$ denote the unit normal vector field, the shape operator, the second fundamental form and its covariant derivative of $Q^{n}$ in $\boldsymbol{R}^{n+1}$, respectively. We note that at generic points $x \in S, \alpha$ restricted to $T_{x}^{*} S$ do not vanish as it is known by the argument in the proof of Theorem 6.4.

To prove our Theorem, we prepare some lemmas.

LEMMA 1. For $x \in S, v \in W^{\perp}$, and $u, u_{1}, u_{2}, u_{3}, u_{4} \in T_{x} S$, the following formulas hold: 
(1) $F(x, u, v)=0$,

(2) $F\left(u_{1}, u_{2}, v\right)+F\left(x, \alpha\left(u_{1}, u_{2}\right), v\right)=0$,

(3) $F\left(\alpha\left(u_{1}, u_{2}\right), u_{3}, v\right)+F\left(\alpha\left(u_{2}, u_{3}\right), u_{1}, v\right)+F\left(\alpha\left(u_{3}, u_{1}\right), u_{2}, v\right)$ $+F\left(x,\left(\bar{\nabla}_{u_{3}} \alpha\right)\left(u_{1}, u_{2}\right), v\right)=0$,

(4) $F\left(\alpha\left(u_{1}, u_{2}\right), \alpha\left(u_{3}, u_{4}\right), v\right)+F\left(\alpha\left(u_{2}, u_{3}\right), \alpha\left(u_{1}, u_{4}\right), v\right)+F\left(\alpha\left(u_{3}, u_{1}\right), \alpha\left(u_{2}, u_{4}\right), v\right)$

$+F\left(\left(\bar{\nabla}_{u_{1}} \alpha\right)\left(u_{2}, u_{3}\right), u_{4}, v\right)+F\left(\left(\bar{\nabla}_{u_{2}} \alpha\right)\left(u_{3}, u_{4}\right), u_{1}, v\right)+F\left(\left(\bar{\nabla}_{u_{3}} \alpha\right)\left(u_{4}, u_{1}\right), u_{2}, v\right)$

$+F\left(\left(\bar{\nabla}_{u_{4}} \alpha\right)\left(u_{1}, u_{2}\right), u_{3}, v\right)-F\left(A_{\alpha\left(u_{3}, u_{1}\right)} u_{4}, u_{2}, v\right)-F\left(A_{\alpha\left(u_{1}, u_{2}\right)} u_{4}, u_{3}, v\right)$

$-F\left(A_{\alpha\left(u_{2}, u_{3}\right)} u_{4}, u_{1}, v\right)+F\left(x,\left(\bar{\nabla}_{u_{4}} \bar{\nabla}_{u_{3}} \alpha\right)\left(u_{1}, u_{2}\right), v\right)=0$.

Proof of Lemma 1. Differentiating $F(x, x, v)=0$, we obtain the first formula (1). Succeedingly differentiating one formula, we obtain next one.

We fix a point $x \in S$ at which $\alpha$ restricted to $T_{x} S$ does not vanish. We define a subspace $W^{\prime}$ in $W^{\perp}$ as follows:

$$
W^{\prime}=\left\{v \in W^{\perp} \mid F\left(x, \xi_{x}, v\right)=0\right\} .
$$

Then we have next lemma.

LEMMA 2. The following formula holds:

$$
F\left(w_{1}, w_{2}, v\right)=0 \text { for any } v \in W^{\prime} \text { and } w_{1}, w_{2} \in \boldsymbol{R} C+W .
$$

Proof of Lemma 2. For $u, u_{1}, u_{2} \in T_{x} S$ and $v \in W^{\prime}$, we have

$$
\begin{array}{llll}
\text { (i) } & F(x, x, v)=0, & \text { (ii) } & F\left(x, \xi_{x}, v\right)=0, \\
\text { (iii) } & F(x, u, v)=0, & \text { (iv) } & F\left(u_{1}, u_{2}, v\right)=0, \\
\text { (v) } & F\left(u, \xi_{x}, v\right)=0, & \text { (vi) } & F\left(\xi_{x}, \xi_{x}, v\right)=0 .
\end{array}
$$

The formula (iii) is exactly Lemma 1 (1) and (iv) is obtained by Lemma 1 (2) and (ii). Since $\alpha$ restricted to $T_{x} S$ does not vanish, by Lemma 1 (3) and (ii) we have (v). Similarly, by Lemma 1 (4) and (ii), (iv) and (v), the formula (vi) holds. Since $W=T_{x} S+\boldsymbol{R} \boldsymbol{\xi}_{x}$, by (iv), (v), and (vi), we have $F\left(w_{1}, w_{2}, v\right)=0$ for $w_{1}, w_{2} \in W$. Moreover by (ii) and (iii), we have $F(x, w, v)=0$ for $w \in W$. Since $x \in S$ is written as $x=C+w$ for some $w \in W$, Lemma 2 holds.

Our important step is to prove that the vector $C$ is zero. We suppose that $C \neq 0$ and will show a contradiction. Moreover we assume that $\operatorname{dim} W^{\prime}=$ $\operatorname{dim} W^{\perp}-1$, where $W^{\prime}$ denotes the subspace of $W^{\perp}$ defined in Lemma 2 (however even if $W^{\prime}=W^{\perp}$, arguments below will hold). To present $W^{\perp}$, we will choose an appropriate basis of $W^{\perp}$. Let $\left\{v_{1}, \cdots, v_{q}\right\}$ be a basis of $W^{\perp}\left(\operatorname{dim} W^{\perp}=q\right)$ such that $\left\{v_{1}, \cdots, v_{q-1}\right\}$ is a basis of $W^{\prime}$. Placing column vectors $v_{1}, \cdots, v_{q}$ of $\boldsymbol{R}^{n+1}$, we obtain an $(n+1, q)$-matrix $P=\left(v_{1} \cdots v_{q}\right)$. We assume that first $(q-1, q-1)$-matrix and first $(q, q)$-matrix in $P$ are both non-singular. If not so, we take a suitable 
permutation $\sigma \in S_{n+1}$ and transform $W, C$ etc. by a linear isometry $T_{\sigma}$. Then our assumption will be satisfied. By change of bases, we may assume that the matrix $P=\left(v_{1} \cdots v_{q}\right)$ has the form:

$$
\left(\begin{array}{cccc}
1 & & 0 & 0 \\
& \ddots & & \vdots \\
0 & & 1 & 0 \\
v_{1}^{q} & \cdots & v_{q-1}^{q} & 1 \\
\cdot & & \cdot & v_{q}^{q+1} \\
\vdots & & \vdots & \vdots \\
v_{1}^{n+1} & \cdots & v_{q-1}^{n+1} & v_{q}^{n+1}
\end{array}\right) .
$$

LEMMA 3. In (6.7), for each $j(j=1, \cdots, q-1), v_{j}^{q}, v_{j}^{q+1}, \cdots, v_{j}^{n+1}$ are zero except at most one and if $v_{j}^{2} \neq 0(i=q, \cdots, n+1)$, then $v_{j}^{2}=-1$. Moreover $W^{\prime}$ is orthogonal to $\boldsymbol{R} C+W$.

Proof of Lemma 3. We prepare some notation. For a vector $v={ }^{t}\left(v^{1}, v^{2}\right.$, $\left.\cdots, v^{n+1}\right) \in \boldsymbol{R}^{n+1}$, we denote by $m_{+}(v)$ and $m_{-}(v)$, the number of positive components $v^{2}$ and the number of negative components $v^{2}$, respectively. We denote by $F_{v}$ a symmetric bilinear form on $\boldsymbol{R}^{n+1}$ defined by $F_{v}(x, y)=F(x, y, v)$, i.e., $F_{v}=\sum_{l=1}^{n+1} v^{2} e_{i}^{*} \otimes e_{i}^{*}$. We note that the dimension of a null subspace with respect to $F_{v}$ is not greater than $n+1-\max \left\{m_{+}(v), m_{-}(v)\right\}$.

ASSERTION 1. For each $j(j=1, \cdots, q-1), v_{j}^{q}, v_{j}^{q+1}, \cdots, v_{j}^{n+1}$ are zero except at most one and if $v_{j}^{2} \neq 0(i=q, \cdots, n+1)$, then $v_{j}^{2}$ is negative.

Proof of Assertion 1. By Lemma 2, $\boldsymbol{R} C+W$ is a null subspace with respect to $F_{v}$ for $v \in W^{\prime}$. Since $C \neq 0$, we have $\operatorname{dim} \boldsymbol{R} C+W=r+2=n+1-(q-1)$. We shall prove our assertion for $j=1$. The other cases are same. Suppose that there exists a positive number in $v_{1}^{q}, v_{1}^{q+1}, \cdots, v_{1}^{n+1}$. We assume that $v_{1}^{2}>0$. We choose sufficiently small positive numbers $\lambda_{2}, \cdots, \lambda_{q-1}$ such that $v_{1}^{2}+\lambda_{2} v_{2}^{2}+\cdots+$ $\lambda_{q-1} v_{q-1}^{2}>0$. Put $v=v_{1}+\lambda_{2} v_{2}+\cdots+\lambda_{q-1} v_{q-1}$. Then we have $m_{+}(v) \geqq q$. Therefore the dimension of a null subspace with respect to $F_{v}$ is not greater than $n+1-q$. It is a contradiction. Next we suppose that there exist two negative numbers in $v_{1}^{q}, v_{1}^{q+1}, \cdots, v_{1}^{n+1}$. We assume that they are $v_{1}^{2}$ and $v_{1}^{j}(q \leqq i<j \leqq n+1)$. Choosing sufficiently small positive numbers $\lambda_{2}, \cdots, \lambda_{q-1}$, we have $v_{1}^{2}-\lambda_{2} v_{2}^{2}-\cdots-\lambda_{q-1} v_{q-1}^{2}<0$ and $v_{1}^{j}-\lambda_{2} v_{2}^{j}-\cdots-\lambda_{q-1} v_{q-1}^{j}<0$. Put $v=v_{1}-\lambda_{2} v_{2}-\cdots-\lambda_{q-1} v_{q-1}$. Then we have $m_{-}(v) \geqq q$. Again it yields a contradiction. Hence our first assertion has been shown. 
Now we take vectors $w_{\imath}(i=1, \cdots, r+1)$ as follows:

$$
w_{\imath}=\left(\begin{array}{c}
v_{1}^{q} v_{q}^{q+\imath}-v_{1}^{q+\imath} \\
v_{2}^{q} v_{q}^{q+\imath}-v_{2}^{q+\imath} \\
\vdots \\
v_{q-1}^{q} v_{q}^{q+\imath}-v_{q-1}^{q+\imath} \\
-v_{q}^{q+\imath} \\
0 \\
\vdots \\
0 \\
1 \\
0 \\
\vdots \\
0
\end{array}\right) \longleftarrow(q+i) \text {-th row. }
$$

Then $\left\{w_{1}, \cdots, w_{r+1}\right\}$ is a basis of $W$. We take a vector $C^{\prime} \in C+W$ which has the form $C^{\prime}={ }^{t}\left(c^{\prime 1}, \cdots, c^{\prime q}, 0, \cdots, 0\right)$. Since $C \neq 0$, we have $C^{\prime} \neq 0$. Evidently $C^{\prime}+W=C+W$.

ASSERTION 2. There exist non-zero ones in $v_{q}^{q+1}, \cdots, v_{q}^{n+1}$.

Proof of Assertion 2. Suppose that $v_{q}^{q+1}=\cdots=v_{q}^{n+1}=0$. For $x \in S$, we have $0=F\left(x, x, v_{q}\right)=\left(x^{q}\right)^{2}$ and hence $x^{q}=0$. Since $S$ is contained in $C^{\prime}+W, 0=$ $\left\langle v_{q}, x-C^{\prime}\right\rangle=-c^{\prime q}$ and hence $c^{\prime q}=0$. Since for $j(1 \leqq j \leqq q-1), 0=F\left(C^{\prime}, C^{\prime}, v_{j}\right)$ $=\left(c^{\prime j}\right)^{2}$, it holds that $c^{\prime \jmath}=0$. Consequently we have $C^{\prime}=0$. It is contrary to $C^{\prime} \neq 0$. Therefore there exist non-zero ones in $v_{q}^{q+1}, \cdots, v_{q}^{n+1}$.

ASSERTION 3. If $v_{j}^{2} \neq 0$ for $j=1, \cdots, q-1$ and $i=q, \cdots, n+1$, then $v_{j}^{\imath}=-1$.

Proof of Assertion 3. For $i=1, \cdots, r+1$, we have

$$
0=F\left(w_{\imath}, w_{\imath}, v_{j}\right)=\left(v_{j}^{q} v_{q}^{q+\imath}-v_{j}^{q+i}\right)^{2}+v_{j}^{q}\left(-v_{q}^{q+i}\right)^{2}+v_{j}^{q+\imath} .
$$

Suppose that $v_{j}^{q+\imath} \neq 0$ for some $i(i=1, \cdots, r+1)$. Then by Assertion 1 , we have $v_{j}^{q}=0$. This, with the formula above, implies that $v_{\jmath}^{q+\imath}\left(v_{\jmath}^{q+\imath}+1\right)=0$ and hence $v_{j}^{q+\imath}=-1$. Suppose that $v_{j}^{q+\imath}=0$ for all $\imath=1, \cdots, r+1$. By Assertion 2, $v_{q}^{q+\imath} \neq 0$ for some $i \quad(i=1, \cdots, r+1)$. From the formula above, it follows that $\left(v_{q}^{q+i}\right)^{2} v_{j}^{q}\left(v_{j}^{q}+1\right)=0$. Therefore we have $v_{j}^{q}=0$ or $v_{j}^{q}=-1$.

ASSERTION 4. For $\jmath=1, \cdots, q-1$, if $v_{j}^{q}=0$, then $c^{\prime \jmath}=0$ and if $v_{j}^{q}=-1$, then $c^{\prime \jmath}=c^{\prime q}$. In particular $W^{\prime}$ is orthogonal to $\boldsymbol{R} C^{\prime}+W=\boldsymbol{R} C+W$.

Proof of Assertion 4. From Lemma 2, it follows that $0=F\left(C^{\prime}, C^{\prime}, v_{\jmath}\right)=$ $\left(c^{\prime \jmath}\right)^{2}+\left(c^{\prime q}\right)^{2} v_{j}^{q}$. Therefore if $v_{j}^{q}=0$, then $c^{\prime \jmath}=0$. Next suppose that $v_{j}^{q}=-1$. We 
choose an integer $\imath$ such that $v_{q}^{q+\imath} \neq 0$. Since $0=F\left(C^{\prime}, w_{\imath}, v_{j}\right)=v_{q}^{q+\imath}\left(c^{\prime q}-c^{\prime j}\right)$, we obtain $c^{\prime \prime}=c^{\prime q}$.

Hence Lemma 3 has been completely proved.

We continue our proof of Theorem 6.7. We use the same notation as Lemma 3 and its proof. We put vectors $w_{\imath}(i=0, \cdots, r+1)$ as follows: $w_{\imath}={ }^{t}\left(-v_{1}^{q+\imath}, \cdots,-v_{q-1}^{q+\imath}, 0, \cdots, 0,1,0, \cdots, 0\right)$, where $v_{j}^{q+\imath}(j=1, \cdots, q-1)$ denote the components of vectors $v$, given in Lemma 3 and $(q+i)$-th row is 1 . Then $\left\{w_{0}, w_{1}, \cdots, w_{r+1}\right\}$ is a basis of $\boldsymbol{R} C+W$. In fact, for each $i, w_{\imath}$ is orthogonal to $v_{1}, \cdots, v_{q-1}$ and hence by Lemma $3, w_{\imath}$ belongs to $\boldsymbol{R} C+W$. For $y \in \boldsymbol{R} C+W$, we write $y=\sum_{\imath=0}^{r+1} y^{2} w_{\imath}$. Then $S$ is a connected component of

$$
(C+W) \cap Q^{n}=\left\{y \in \boldsymbol{R} C+W \mid y^{0}+\sum_{i=1}^{r+1} v_{q}^{q+\imath} y^{\imath}=c^{\prime q}, \sum_{i=0}^{r+1} m_{i}\left(y^{i}\right)^{3}=1\right\} .
$$

Here $m_{\imath}(i=0, \cdots, r+1)$ denote positive integers defined by $m_{\imath}=1+$ the number of $\left\{j \mid v_{J}^{q+\imath}=-1\right\}$. Moreover it holds that $F\left(y, y, v_{q}\right)=0$ for all $y \in S$, i.e., $\left(y^{0}\right)^{2}+\sum_{i=1}^{r+1} v_{q}^{q+\imath}\left(y^{i}\right)^{2}=0$. From now on, we simply write $v^{2}(i=1, \cdots, r+1)$ and $c$ for $v_{q}^{q+\imath}$ and $c^{\prime q}$, respectively. We note that $c=c^{\prime q}$ is not zero. In fact, if $c^{\prime q}=0$, then by Assertion 4 in the proof of Lemma 3, we have $C^{\prime}=0$ and it is contrary to our assumption. We define functions $f, g$, and $h$ on $\boldsymbol{R} C+W$ by $f(y)=\sum_{\imath=0}^{r+1} m_{i}\left(y^{i}\right)^{3}, \quad g(y)=y^{0}+\sum_{l=1}^{r+1} v^{2} y^{2}, \quad$ and $h(y)=\left(y^{0}\right)^{2}+\sum_{l=1}^{r+1} v^{2}\left(y^{i}\right)^{2}$, respectively.

As is shown in Assertion 2, there exist non-zero ones in $v^{1}, \cdots, v^{r+1}$. We shall show that there exist negative ones in them. Suppose that $v^{1}, \cdots, v^{r+1}$ are all non-negative. Since $h(y)=0$ for $y \in S$, we have $g(y)=0$. It is contrary to $g(y)=c \neq 0$ for $y \in S$. Therefore there exist negative ones. So we assume that $v^{1}$ is negative. We define a projection $\pi$ of $\boldsymbol{R} C+W$ onto the subspace $V$ linearly spanned by $w_{2}, \cdots, w_{r+1}$ as follows: $\pi(y)=\pi\left(\sum_{\imath=0}^{r+1} y^{\imath} w_{\imath}\right)=\sum_{\imath=2}^{r+1} y^{2} w_{\imath}$ for $y \in \boldsymbol{R} C+W$. It is easily seen that $\pi$ restricted to $(C+W) \cap Q^{n}$ is a homeomorphism onto $V$. Therefore $(C+W) \cap Q^{n}$ is connected and hence coincides with $S$. Next we shall show that there exist non-zero ones in $v^{2}, \cdots, v^{r+1}$. Suppose that $v^{2}=\cdots=v^{r+1}=0$. We put $y=c w_{0}+y^{2} w_{2}$, where $y^{2}=\sqrt[3]{\left(1-m_{0} c^{3}\right) / m_{2}}$. Then $y$ belongs to $S$ and $h(y)=c^{2} \neq 0$. It is a contradiction. Therefore there exist non-zero ones in $v^{2}, \cdots, v^{r+1}$. So we assume that $v^{2} \neq 0$.

We take $y^{0}$ and $y^{1}$ which satisfy $y^{0}+v^{1} y^{1}=c$ and $m_{0}\left(y^{0}\right)^{3}+m_{1}\left(y^{1}\right)^{3}=1$ and put $y=y^{0} w_{0}+y^{1} w_{1}$. Then $y$ belongs to $S$. Since $h \equiv 0$ on $S$, it holds that $d h=\lambda d f+\mu d g$ in $T_{y}^{*}(\boldsymbol{R} C+W)$ for some $\lambda, \mu \in \boldsymbol{R}$. At $y=y^{0} w_{0}+y^{1} w_{1}$, we have $d f=3 m_{0}\left(y^{0}\right)^{2} d y^{0}+3 m_{1}\left(y^{1}\right)^{2} d y^{1}, \quad d g=d y^{0}+\sum_{\imath=1}^{r+1} v^{2} d y^{2}$ and $d h=2 y^{0} d y^{0}+2 v^{1} y^{1} d y^{1}$. Comparing coefficients of $d y^{2}$ in both sides, we obtain $\mu=0$. Since $d h=\lambda d f$, it follows that $2 y^{0}=3 \lambda m_{0}\left(y^{0}\right)^{2}$ and $2 v^{1} y^{1}=3 \lambda m_{1}\left(y^{1}\right)^{2}$. Since $h(y)=0$, we have $0=2\left\{\left(y^{0}\right)^{2}+v^{1}\left(y^{1}\right)^{2}\right\}=3 \lambda\left\{m_{0}\left(y^{0}\right)^{3}+m_{1}\left(y^{1}\right)^{3}\right\}=3 \lambda$ and hence $\lambda=0$. From this it follows that $d h=0$ and hence $y^{0}=y^{1}=0$. It is a contradiction. Consequently it has been proved that the vector $C$ is zero. 
By the argument above, it follows that $S$ is a connected component of $W \cap Q^{n}$, where $W$ is an $(r+1)$-dimensional linear subspace of $\boldsymbol{R}^{n+1}$. Since $F(x, x, v)=0$ for any $x \in S$ and $v \in W^{\perp}$, we have $F\left(w_{1}, w_{2}, v\right)=0$ for any $w_{1}, w_{2}$ $\in W$ and $v \in W^{\perp}$. By the same consideration as Lemma 3, we see that there exists a basis $\left\{v_{1}, \cdots, v_{q}\right\}$ of $W^{\perp}$ which has the following form (up to a linear isometry $\left.T_{\sigma}, \sigma \in S_{n+1}\right)$ :

$$
\left(v_{1} \cdots v_{q}\right)=\left(\begin{array}{ccc}
1 & & 0 \\
0 & \ddots & 1 \\
v_{1}^{q+1} & \cdots & v_{q}^{q+1} \\
\vdots & & \vdots \\
v_{1}^{n+1} & \cdots & v_{q}^{n+1}
\end{array}\right)
$$

Here for each $j(j=1, \cdots, q), v_{j}^{q+1}, \cdots, v_{j}^{n+1}$ are zero except at most one and if $v_{j}^{2} \neq 0(i=q+1, \cdots, n+1)$, then $v_{j}^{2}=-1$. Therefore it has been proved that $S$ is congruent to $S_{k_{1}, \cdots, k_{l}}$ in $Q^{m}$ for some $m \leqq n$.

On case (2) in Theorem 6.7 the following holds:

PROPOSITION 6.8. The dimension of an affine subspace contained in $Q^{n}$ is not greater than $n / 2$. When $n=2 q$, a q-dimensional affine subspace contained in $Q^{n}$ is congruent to the following affine subspace $S$ by a linear transformation $T_{\sigma}$, $\sigma \in S_{n+1}:$

$$
S=\left\{x={ }^{t}\left(x^{1}, \cdots, x^{n+1}\right) \in \boldsymbol{R}^{n+1} \mid x^{\imath}+x^{q+\imath}=0(i=1, \cdots, q), x^{n+1}=1\right\}
$$

Proof. Let $S$ be an $r$-dimensional affine subspace of $\boldsymbol{R}^{n+1}$ which is contained in $Q^{n}$ and $W$ be an $r$-dimensional subspace which corresponds to the tangent space $T_{x} S$ at $x \in S$. Then the following formulas hold:

$$
\begin{aligned}
& \text { (i) } F(x, x, x)=1, \quad \text { (ii) } F\left(x, x, w_{1}\right)=0 \text {, } \\
& \text { (iii) } F\left(x, w_{2}, w_{1}\right)=0, \quad \text { (iv) } F\left(w_{3}, w_{2}, w_{1}\right)=0
\end{aligned}
$$

for $w_{1}, w_{2}, w_{3} \in W$.

We use the same notation as Lemma 3 in Theorem 6.7. The formulas (ii), (iii), and (iv) imply that an $r+1$-dimensional linear subspace $\boldsymbol{R} x+W$ is a null subspace with respect to $F_{w}$ for any $w \in W$. We may take a basis $\left\{v_{1}, \cdots, v_{r}\right\}$ of $W$ which has the following form (up to a linear transformation $T_{\sigma}, \sigma \in S_{n+1}$ ):

$$
\left(v_{1} \cdots v_{r}\right)=\left(\begin{array}{ccc}
1 & & 0 \\
0 & \ddots & 1 \\
v_{1}^{r+1} & \cdots & v_{r}^{r+1} \\
\vdots & & \vdots \\
v_{1}^{n+1} & \cdots & v_{r}^{n+1}
\end{array}\right)
$$


We put $v=v_{1}+\cdots+v_{r}$. Since $m_{+}(v) \geqq r$, the dimension of a null subspace with respect to $F_{v}$ is not greater than $n+1-r$. Therefore we have $r+1 \leqq n+1-r$ and hence $r \leqq n / 2$. Thus the first part of Proposition 6.8 has been proved.

From now on we assume that $n=2 q$ and that $S$ is a $q$-dimensional affine subspace of $\boldsymbol{R}^{n+1}$ which is contained in $Q^{n}$. Let $\left\{v_{1}, \cdots, v_{q}\right\}$ be a basis of $W$ which has the same form as (6.8). By the same argument as Lemma 3 in Theorem 6.7, we see that for each $j(\jmath=1, \cdots, q), v_{j}^{q+1}, \cdots, v_{j}^{n+1}$ are zero except at most one. Since $0=F\left(v_{\jmath}, v_{\jmath}, v_{j}\right)=1+\sum_{\imath=1}^{q+1}\left(v_{j}^{q+i}\right)^{3}$, there exists only one $v_{\jmath}^{q+\imath}$ such that $v_{j}^{q+\imath}=-1$. For $k \neq j$, we have $0=F\left(v_{k}, v_{k}, v_{j}\right)=v_{j}^{q+\imath}\left(v_{k}^{q+i}\right)^{2}=-\left(v_{k}^{q+i}\right)^{2}$ and hence $v_{k}^{q+\imath}=0$. Therefore transforming by $T_{q}, \sigma \in S_{n+1}$, if necessary, we can take a basis $\left\{v_{1}, \cdots, v_{q}\right\}$ of $W$ which has the following form:

$$
\left(v_{1} \cdots v_{q}\right)=\left(\begin{array}{ccc}
1 & & 0 \\
0 & \ddots & 1 \\
-1 & & 0 \\
0 & \ddots & -1 \\
0 & \cdots & 0
\end{array}\right) \text {. }
$$

Since $F\left(x, v_{\jmath}, v_{j}\right)=0$, we have $x^{j}+x^{q+\jmath}=0 \quad(j=1, \cdots, q)$. Therefore the vector ${ }^{t}(0, \cdots, 0,1)$ belongs to $S=x+W$. Consequently the second part of Proposition 6.8 has been proved.

\section{Totally geodesic surfaces of 3-dimensional Lie groups with left invariant Riemannian metric}

Let $G$ be a Lie group with left invariant Riemannian metric and let $g$ be the corresponding Lie algebra with an inner product $\langle$,$\rangle . We want to clarify$ various properties on totally geodesic submanifolds in $G$. In this paper we will study the simplest case, i.e., 2-dimensional submanifolds in 3-dimensional Lie groups. From now on we denote by $G$ a 3-dimensional Lie group. We reduce the classification problem of totally geodesic surfaces of $G$ to the following problem: Classify 2-dimensional subspaces in $T_{e} G$ which are tangent spaces of totally geodesic surfaces in $G$. Here $e$ denotes the identity of $G$. As usual, we identify $T_{e} G$ with $g$. In this paper we specialize to the unimodular case and state our results.

First we recall the classification of 3-dimensional unimodular Lie groups following J. Milnor [10]. Let $g$ be a 3-dimensional unimodular Lie algebra with an inner product $\langle$,$\rangle . Then there exists an orthonormal basis e_{1}, e_{2}, e_{3}$ for which the bracket operation is given as follows:

$$
\left[e_{2}, e_{3}\right]=\lambda_{1} e_{1}, \quad\left[e_{3}, e_{1}\right]=\lambda_{2} e_{2}, \quad\left[e_{1}, e_{2}\right]=\lambda_{3} e_{3} .
$$


According to signs of $\lambda_{1}, \lambda_{2}, \lambda_{3}$, six kinds of Lie algebras are obtained. The following table is due to J. Milnor $[10]$ :

Table 1.

\begin{tabular}{c|c}
\hline Signs of $\lambda_{1}, \lambda_{2}, \lambda_{3}$ & Associated Lie group \\
\hline,,+++ & $S U(2)$ or $S O(3)$ \\
,,++- & $S L(2, \boldsymbol{R})$ or $O(1,2)$ \\
,,++ 0 & $E(2)$ \\
,,+- 0 & $E(1,1)$ \\
$+, 0,0$ & Heisenberg group \\
$0,0,0$ & $\boldsymbol{R}^{3}$ \\
\hline
\end{tabular}

Now we give the formulas of the Riemannian connection $\nabla$ and the curvature properties, which are due to [10]. For convenience, we define numbers $\mu_{2}$ by $\mu_{2}=1 / 2\left(\lambda_{1}+\lambda_{2}+\lambda_{3}\right)-\lambda_{2}(i=1,2,3)$.

LEMMA 7.1. Let $e_{1}, e_{2}, e_{3}$ be an orthonormal basis of $(\mathrm{g},\langle\rangle$,$) chosen as (7.1).$

(1) The Riemannian connection $\nabla$ is given with respect to $e_{1}, e_{2}, e_{3}$ as follows:

$$
\begin{array}{ll}
\nabla_{e_{1}} e_{1}=\nabla_{e_{2}} e_{2}=\nabla_{e_{3}} e_{3}=0 \\
\nabla_{e_{1}} e_{2}=\mu_{1} e_{3}, & \nabla_{e_{1}} e_{3}=-\mu_{1} e_{2} \\
\nabla_{e_{2}} e_{1}=-\mu_{2} e_{3}, & \nabla_{e_{2} e_{3}}=\mu_{2} e_{1} \\
\nabla_{e_{3}} e_{1}=\mu_{3} e_{2}, & \nabla_{e_{3}} e_{2}=-\mu_{3} e_{1} .
\end{array}
$$

(2) The orthonormal basis $e_{1}, e_{2}, e_{3}$ diagonalizes the Ricci curvature tensor and the principal Ricci curvatures are given by

$$
\rho\left(e_{1}, e_{1}\right)=2 \mu_{2} \mu_{3}, \quad \rho\left(e_{2}, e_{2}\right)=2 \mu_{1} \mu_{3}, \quad \rho\left(e_{3}, e_{3}\right)=2 \mu_{1} \mu_{2} .
$$

(3) The covariant derivative of the curvature tensor $R$ is given as follows:

$$
\begin{array}{ll}
\left(\nabla_{e_{1}} R\right)\left(e_{1}, e_{2}\right) e_{1}=2 \mu_{1}^{2}\left(\mu_{2}-\mu_{3}\right) e_{3}, & \left(\nabla_{e_{1}} R\right)\left(e_{1}, e_{3}\right) e_{1}=2 \mu_{1}^{2}\left(\mu_{2}-\mu_{3}\right) e_{2} \\
\left(\nabla_{e_{1}} R\right)\left(e_{1}, e_{2}\right) e_{3}=-2 \mu_{1}^{2}\left(\mu_{2}-\mu_{3}\right) e_{1}, & \left(\nabla_{e_{1}} R\right)\left(e_{1}, e_{3}\right) e_{2}=-2 \mu_{1}^{2}\left(\mu_{2}-\mu_{3}\right) e_{1} \\
\left(\nabla_{e_{2}} R\right)\left(e_{2}, e_{1}\right) e_{2}=2 \mu_{2}^{2}\left(\mu_{3}-\mu_{1}\right) e_{3}, & \left(\nabla_{e_{2}} R\right)\left(e_{2}, e_{3}\right) e_{2}=2 \mu_{2}^{2}\left(\mu_{3}-\mu_{1}\right) e_{1} \\
\left(\nabla_{e_{2}} R\right)\left(e_{2}, e_{1}\right) e_{3}=-2 \mu_{2}^{2}\left(\mu_{3}-\mu_{1}\right) e_{2}, & \left(\nabla_{e_{2}} R\right)\left(e_{2}, e_{3}\right) e_{1}=-2 \mu_{2}^{2}\left(\mu_{3}-\mu_{1}\right) e_{2} \\
\left(\nabla_{e_{3}} R\right)\left(e_{3}, e_{1}\right) e_{3}=2 \mu_{3}^{2}\left(\mu_{1}-\mu_{2}\right) e_{2}, & \left(\nabla_{e_{3}} R\right)\left(e_{3}, e_{2}\right) e_{3}=2 \mu_{3}^{2}\left(\mu_{1}-\mu_{2}\right) e_{1} \\
\left(\nabla_{e_{3}} R\right)\left(e_{3}, e_{1}\right) e_{2}=-2 \mu_{3}^{2}\left(\mu_{1}-\mu_{2}\right) e_{3}, & \left(\nabla_{e_{3}} R\right)\left(e_{3}, e_{2}\right) e_{1}=-2 \mu_{3}^{2}\left(\mu_{1}-\mu_{2}\right) e_{3}
\end{array}
$$

the others $\left(\nabla_{e_{i}} R\right)\left(e_{\jmath}, e_{k}\right) e_{l}=0$. 
Proof. (1) and (2) are found in [10] and (3) is obtained by the straightforward computation.

Let $\operatorname{Aut}(\mathrm{g})$ and $O(\mathfrak{g} ;\langle\rangle$,$) be the group consisting of automorphisms of the$ Lie algebra $g$ and that of orthogonal transformations of $(g,\langle\rangle$,$) , respectively.$ For $\Phi \in \operatorname{Aut}(\mathrm{g})$, we take the (local) automorphism $\varphi$ of the Lie group $G$ such that $\varphi_{* e}=\Phi$. Moreover if $\Phi \in O(g ;\langle\rangle$,$) , then \varphi$ is an (local) isometry of $G$ with the left invariant Riemannian metric. Therefore a 2-dimensional subspace $V$ in $T_{e} G \cong g$ is the tangent space of a totally geodesic surface in $G$ if and only if so is $\Phi(V)$. We will classify such subspaces up to the action of $\operatorname{Aut}(\mathrm{g}) \cap O(\mathrm{~g} ;\langle\rangle$,$) .$

THEOREM 7.2. Let $G$ be a 3-dimensional unimodular Lie group with left invariant Riemannian metric and let $e_{1}, e_{2}, e_{3}$ be an orthonormal basis of $(\mathfrak{g} ;\langle\rangle$, chosen as (7.1). Then totally geodesic surfaces of $G$ are classified as the following table: to describe totally geodesic surfaces, we list up 2-dimensional subspaces in $T_{e} G \cong g$ which are the tangent spaces of totally geodesic surfaces. We remark that the signs of $\lambda_{1}, \lambda_{2}, \lambda_{3}$ in the table below are chosen as Table 1.

\begin{tabular}{|c|c|c|c|}
\hline$G$ & metric & t.g. surfaces & remark \\
\hline$S U(2)$ & $\begin{array}{l}\lambda_{1}=\lambda_{2}=\lambda_{3} \\
\text { the others }\end{array}$ & $\begin{array}{l}\text { all 2-dim subspaces } \\
\text { none }\end{array}$ & $(1)$ \\
\hline$S L(2, \boldsymbol{R})$ & $\begin{array}{l}\lambda_{1}>\lambda_{2}-\lambda_{3} \\
\lambda_{1}=\lambda_{2}-\lambda_{3} \\
\lambda_{1}<\lambda_{2}-\lambda_{3}\end{array}$ & $\begin{array}{c}\text { none } \\
\left\{\cos \theta e_{1}+\sin \theta e_{3}, e_{2}\right\}_{R} \\
\left(\tan ^{2} \theta=-\frac{\lambda_{3}}{\lambda_{1}}\right) \\
\text { none }\end{array}$ & $(2)$ \\
\hline$E(2)$ & $\begin{array}{l}\lambda_{1}>\lambda_{2} \\
\lambda_{1}=\lambda_{2}\end{array}$ & $\begin{array}{c}\text { none } \\
\text { all 2-dim subspaces }\end{array}$ & (3) \\
\hline$E(1,1)$ & $\begin{array}{l}\lambda_{1} \neq-\lambda_{2} \\
\lambda_{1}=-\lambda_{2}\end{array}$ & $\begin{array}{c}\text { none } \\
\left\{\frac{1}{\sqrt{2}}\left(e_{1}+e_{2}\right), e_{3}\right\}_{\boldsymbol{R}} \text { and } \\
\left\{\frac{1}{\sqrt{2}}\left(e_{1}-e_{2}\right), e_{3}\right\}_{\boldsymbol{R}}\end{array}$ & (4) \\
\hline Heisenberg $g r$. & & none & \\
\hline$R^{3}$ & & all 2-dim subspaces & (5) \\
\hline
\end{tabular}

Remarks :

(1) This metric is of positive constant sectional curvature.

(2) These subspaces are equivalent under the action of $\operatorname{Aut}(\mathrm{g}) \cap O(\mathrm{~g} ;\langle\rangle$,$) .$ They are Lie subalgebras of $\mathrm{g}$. 
(3) This metric is flat.

(4) These two subspaces are equivalent under the action of $\operatorname{Aut}(\mathrm{g}) \cap O(\mathrm{~g} ;\langle\rangle$,$) .$ They are Lie subalgebras of $\mathrm{g}$.

(5) This metric is flat.

Proof. We shall prove our computation results when $G=S L(2, \boldsymbol{R})$. The other cases are similar. We may assume that $\lambda_{1} \geqq \lambda_{2}$ without loss of generality. Since $\lambda_{3}<0$, we have $\mu_{1}=1 / 2\left(-\lambda_{1}+\lambda_{2}+\lambda_{3}\right)<0, \mu_{3}=1 / 2\left(\lambda_{1}+\lambda_{2}-\lambda_{3}\right)>0$, and $\mu_{2}<\mu_{3}$. The sign of $\mu_{2}$ is uncertain. We consider the following three cases:

Case I. $\mu_{2}>0$.

Case II. $\mu_{2}=0$.

Case III. $\mu_{2}<0$.

Case I. By Lemma 7.1 (2), the eigenvalues of Ricci curvature tensor are mutually distinct. Therefore by Proposition 4.6 curvature-invariant subspaces are $\left\{e_{1}, e_{2}\right\}_{R},\left\{e_{2}, e_{3}\right\}_{R}$, and $\left\{e_{1}, e_{3}\right\}_{R}$. Since $\left(\nabla_{e_{1}} R\right)\left(e_{1}, e_{2}\right) e_{1}=2 \mu_{1}^{2}\left(\mu_{2}-\mu_{3}\right) e_{3}$, there does not exist a totally geodesic surface whose tangent space at $e$ is $\left\{e_{1}, e_{2}\right\}_{R}$. For subspaces $\left\{e_{2}, e_{3}\right\}_{R}$ and $\left\{e_{1}, e_{3}\right\}_{R}$, the same holds. Hence in this case there isn't a totally geodesic surface.

Case II. In this case, we have $\rho\left(e_{1}, e_{1}\right)=\rho\left(e_{3}, e_{3}\right)=0$ and $\rho\left(e_{2}, e_{2}\right)<0$. Therefore curvature-invariant subspaces are $\left\{e_{1}, e_{3}\right\}_{R}$ and $\left\{\cos \theta e_{1}+\sin \theta e_{3}, e_{2}\right\}_{R}$ for $\theta \in \boldsymbol{R}$.

Case $1,\left\{e_{1}, e_{3}\right\}_{R}$. Since $\left(\nabla_{e_{1}} R\right)\left(e_{1}, e_{3}\right) e_{1}=-2 \mu_{1}^{2} \mu_{3} e_{2}$, there does not exist a totally geodesic surface whose tangent space at $e$ is $\left\{e_{1}, e_{3}\right\}_{R}$.

Case 2, $\left\{\cos \theta e_{1}+\sin \theta e_{3}, e_{2}\right\}_{R}$. We put $f=\cos \theta e_{1}+\sin \theta e_{3}$. Making use of Lemma 7.1 (3), we have

$$
\left(\nabla_{f} R\right)\left(f, e_{2}\right) f=2 \mu_{1} \mu_{3}\left(\cos ^{2} \theta \mu_{1}+\sin ^{2} \theta \mu_{3}\right)\left(\sin \theta e_{1}-\cos \theta e_{3}\right) .
$$

Therefore if $\cos ^{2} \theta \mu_{1}+\sin ^{2} \theta \mu_{3} \neq 0$, there does not exist a totally geodesic surface whose tangent space at $e$ is $\left\{f, e_{2}\right\}_{R}$. From now on we choose $\theta$ which satisfies $\tan ^{2} \theta=-\mu_{1} / \mu_{3}=-\lambda_{3} / \lambda_{1}$. In this case, we put $\mathfrak{h}=\left\{f, e_{2}\right\}_{R}$. Then $\mathfrak{h}$ is a Lie subalgebra of $g$. In fact we have

$$
\left[f, e_{2}\right]=-\lambda_{1} \sin \theta e_{1}+\lambda_{3} \cos \theta e_{3}=-\lambda_{1} \tan \theta f .
$$

Let $H$ be a connected Lie subgroup of $G$ which corresponds to $\mathfrak{h}$. Then $H$ is totally geodesic in $G$. In fact we have $\nabla_{f} f=\sin \theta \cos \theta\left(-\mu_{1}+\mu_{3}\right) e_{2}, \nabla_{e_{2}} f=0$, and $\nabla_{e_{2}} e_{2}=0$.

Case III. We devide this case into the following two subcases:

Case $1 . \quad \lambda_{1}>\lambda_{2}$.

Case 2. $\lambda_{1}=\lambda_{2}$.

Case 1. Since $\mu_{1}-\mu_{2}=\lambda_{2}-\lambda_{1}<0$, the eigenvalues of Ricci curvature tensor are mutually distinct. Similarly to Case I, we see that there does not exist a totally geodesic surface. 
Case 2. In this case, we have $\rho\left(e_{1}, e_{1}\right)=\rho\left(e_{2}, e_{2}\right)<0$ and $\rho\left(e_{3}, e_{3}\right)>0$. Therefore curvature-invariant subspaces are $\left\{e_{1}, e_{2}\right\}_{R}$ and $\left\{\cos \theta e_{1}+\sin \theta e_{2}, e_{3}\right\}_{R}$ for $\theta \in \boldsymbol{R}$. Moreover it is easily seen that

$$
\left\{\left(\begin{array}{ccc}
\cos \alpha & -\sin \alpha & 0 \\
\sin \alpha & \cos \alpha & 0 \\
0 & 0 & 1
\end{array}\right) \mid \alpha \in \boldsymbol{R}\right\} \subset \operatorname{Aut}(\mathrm{g}) \cap O(\mathrm{~g} ;\langle,\rangle) .
$$

Therefore it is sufficient to consider the subspaces $\left\{e_{1}, e_{2}\right\}_{R}$ and $\left\{e_{1}, e_{3}\right\}_{R}$. Since $\left(\nabla_{e_{1}} R\right)\left(e_{1}, e_{2}\right) e_{1}=2 \mu_{1}^{2}\left(\mu_{2}-\mu_{3}\right) e_{3}$ and $\left(\nabla_{e_{1}} R\right)\left(e_{1}, e_{3}\right) e_{1}=2 \mu_{1}^{2}\left(\mu_{2}-\mu_{3}\right) e_{2}$, there does not exist a totally geodesic surface whose tangent space at $e$ is $\left\{e_{1}, e_{2}\right\}_{R}$ or $\left\{e_{1}, e_{3}\right\}_{R}$.

Observations of Theorem 7.2. (1) There do not exist totally geodesic surfaces in almost all 3-dimensional Lie groups with left invariant Riemannian metrics.

(2) Except the cases of constant sectional curvature, totally geodesic surfaces are Lie subgroups. In particular, the equivariance of such immersions holds.

(3) After we classified totally geodesic surfaces, we see that given a 2-dimensional subspace $V$ in $T_{e} G$ there is a totally geodesic surface whose tangent space at $e$ is $V$ if and only if $R(x, y) z \in V$ and $\left(\nabla_{u} R\right)(x, y) z \in V$ for $u, x, y, z \in V$. That is, we can conclude that there exists or not a totally geodesic submanifold by the data of $R$ and $\nabla R$.

\section{REFERENCES}

[1] J.P. Bourguignon AND H. KARCHER, Curvature operators: pinching estimates and geometric examples, Ann. Sci. École Norm. Sup., 11 (1978), 71-92.

[2] B.Y. CHEN, Classification of totally umbilical submanifolds in symmetric spaces, J. Austral. Math. Soc., 30 (1980), 129-136.

[3] B. Y. CHEN AND T. NAGANO, Totally geodesic submanifolds of symmetric spaces II, Duke Math. J., 45 (1978), 405-425.

[4] C. Chevalley, Theory of Lie Groups, Princeton University Press, 1946.

[5] P. Dombrowski, Differentiable maps into riemannian manifolds of constant stable osculating rank I, J. Reine Angew. Math., 274/275 (1975), 310-341.

[6] J. ERBACHER, Reducation of the codimension of an isometric immersion, J. Differential Geom., 5 (1971), 333-340.

[7] R. HERmann, Existence in the large of totally geodesic submanifolds of Riemannian spaces, Bull. Amer. Math. Soc., 66 (1960), 59-61.

[8] S. Kobayashi and K. Nomizu, Foundations of Differential Geometry I, II, Interscience publishers, 1963, 1969.

[9] R.S. KulKarni, Curvature structures and conformal transformations, J. Differential Geom., 4 (1970), 425-451.

[10] J. Milnor, Curvatures of left invariant metrics on Lie groups, Adv. Math., 21 (1976), 293-329. 
[11] H. RECKZIEGEL, A class of distinguished isometric immersions with parallel second fundamental form, Resultate Math., 6 (1983), 56-63.

[12] M. SpIVAK, A Comprehensive Introduction to Differential Geometry Vol. 3, Publish or Perish Inc., 1975.

[13] K. Tojo, Totally geodesic submanifolds of naturally reductive homogeneous spaces, Tsukuba J. Math., 20 (1996), 181-190.

[14] K. Tojo, Totally geodesic hypersurfaces of normal homogeneous spaces, to appear.

[15] J.P. Wolf, The geometry and structure of isotropy irreducible homogeneous spaces, Acta. Math., 120 (1968), 59-148.

Department of Mathematics

OCHANOMIZU UNIVERSITY

TOKYO 112

JAPAN 
